\title{
Universality for conformally invariant intersection exponents
}

Received June 4, 1999 / final version received June 20, 2000

Published online September 7, 2000 - (C) Springer-Verlag \& EMS 2000

\begin{abstract}
We construct a class of conformally invariant measures on sets (or paths) and we study the critical exponents called intersection exponents associated to these measures. We show that these exponents exist and that they correspond to intersection exponents between planar Brownian motions. More precisely, using the definitions and results of our paper [27], we show that any set defined under such a conformal invariant measure behaves exactly as a pack (containing maybe a non-integer number) of Brownian motions as far as all intersection exponents are concerned. We show how conjectures about exponents for two-dimensional self-avoiding walks and critical percolation clusters can be reinterpreted in terms of conjectures on Brownian exponents.
\end{abstract}

\section{Introduction}

Many critical two-dimensional lattice models in statistical physics, for instance self-avoiding walks (polymers) and critical percolation, are conjectured to have continuum limits that are in some sense conformally invariant. This conformal invariance can be exploited to obtain predictions for critical exponents, and these conjectures are consistent with values obtained by Monte Carlo simulation. It appears very difficult to make these arguments rigorous, yet they do suggest that conformal invariance is crucial in understanding the behavior of these systems.

A mathematically analogous system that does not a priori seem to correspond directly to a model from statistical physics is non-intersecting Brownian motions or non-intersecting simple random walks, i.e., random paths that can have self-intersections, but such that different paths do not intersect. This is an example where the critical exponents are mathematically well-defined

G.F. Lawler: Department of Mathematics, Duke University, Box 90320, Durham,

NC 27708-0320, USA; e-mail: jose @ math.duke.edu

Research supported by the National Science Foundation

W. Werner: Département de Mathématiques, Université Paris-Sud, Bât. 425,

91405 Orsay cedex, France; e-mail: wendelin.werner@math.u-psud.fr

Mathematics Subject Classification (1991): 81T40, $60 \mathrm{~J} 65$ 
(and the exponents for the continuum limit, non-intersecting Brownian motions, turn out the same as the exponents for the lattice model, non-intersecting random walks) and conformal invariance can be used to prove some theorems about the exponents. However, while exact values for the exponents have been conjectured, these values have not been verified rigorously.

Suppose that $n+p$ independent planar Brownian motions $\beta^{1}, \cdots, \beta^{n}$ and $\gamma^{1}, \cdots, \gamma^{p}$ are started from points $\beta_{0}^{1}=\cdots=\beta_{0}^{n}=(0,0)$ and $\gamma_{0}^{1}=\cdots=\gamma_{0}^{p}=(1,0)$ in the complex plane, and consider the probability that for all $j \leq n$ and $l \leq p$, the paths of $\beta^{j}$ up to time $t$ and of $\gamma^{l}$ up to time $t$ do not intersect; more precisely:

$$
f_{n, p}(t)=P\left(\bigcup_{j=1}^{n} \beta^{j}[0, t] \cap \bigcup_{l=1}^{p} \gamma^{l}[0, t]=\emptyset\right) .
$$

It is easy to see that this probability decays as $t \rightarrow \infty$ roughly like a power of $t$. The $(n, p)$-intersection exponent $\xi(n, p)$ is defined as being twice this power, i.e.,

$$
f_{n, p}(t) \approx t^{-\xi(n, p) / 2}, \quad t \rightarrow \infty
$$

(we write $a(t) \approx b(t)$ and say $a(t)$ is logarithmically asymptotic to $b(t)$ if $\log (a(t)) / \log (b(t)) \rightarrow 1)$. We call $\xi(n, p)$ the intersection exponent between one packet of $n$ Brownian motions and one packet of $p$ Brownian motions (for a list of references on Brownian intersection exponents, see [27]). In [27], we showed that there is a natural and rigorous way to generalize the definition of intersection exponents between packs of Brownian motions to the case where each pack of Brownian motions is the union of a "non-integer number" of paths; for instance, one can define the exponent $\xi\left(\alpha, \alpha^{\prime}\right)$ for any reals $\alpha, \alpha^{\prime} \geq 1$.

In the present paper, we define a class of measures on sets (or paths) that have a certain conformal invariance property. This class of measures contains Brownian paths (and unions of independent Brownian paths and collections of non-intersecting Brownian paths). The nonrigorous predictions of conformal field theory suggest that other 2-dimensional models may (in the scaling limit) fit into this category, or at least have the same exponents as measures in this category. Examples are: self-avoiding walks (or, perhaps, pairs of non-intersecting self-avoiding walks) and critical percolation clusters.

The main results of the present paper can be loosely speaking described as follows. Take a measure $\mu$ belonging to our conformally invariant class of measures. Then, there exists a deterministic number $\alpha=\alpha(\mu)$, such that as far as intersection exponents are concerned, the path defined under 
the measure $\mu$ behaves as a pack of $\alpha$ Brownian motions. For instance, the intersection exponent between $N$ independent copies of the paths defined under $\mu$ is simply the intersection exponent between $N$ packs of $\alpha$ Brownian motions each.

This leads to the three following open questions:

- Are there really other (i.e. non-Brownian) measures in our class of conformally invariant measures?

- Do the measures associated to two-dimensional self-avoiding walks and critical percolation clusters converge (in the scaling limit) to measures in the class (or at least in the same "universality class" as measures in this class)?

- And of course: How could we prove the exact conjectures for the value of the exponents?

Our results are consistent with physicists' predictions for values of exponents and shed new light on why the conjectures for series of intersection exponents have the same general structure (a quadratic function of the number of packs) in the different models. Also, they indicate that if one can prove rigorously the values of the exponents for Brownian motion (or any other measure in our class), one gets all other exponents for free. In terms of nonrigorous conformal field theory (see, e.g., [17, Chapt. 9]), our results could be formulated as follows: any intersection exponent corresponding to a model with central charge equal to zero can be interpreted as an intersection exponent between Brownian motions. For instance, we give a nonrigorous justification of the fact that the Brownian frontier (the frontier of a connected component of the complement of a Brownian path), the "scaling limit" of self-avoiding walk, and that of the outer frontier of a macroscopic critical percolation cluster have the same dimension (and multifractal spectrum). We also derive (nonrigorously) some (nonrigorously) well-known exponents for self-avoiding walk in two dimensions using the predictions for Brownian exponents. In particular, we discuss why it is natural to consider a self-avoiding walk as " $5 / 8$ of a Brownian motion", and use this and the Brownian conjectures to derive the intersection exponents for self-avoiding walk. See [12] for a similar, but somewhat different, nonrigorous argument that $\alpha=5 / 8$ corresponds to self-avoiding walk.

The paper is structured as follows. In the next section, we define the class of conformally invariant measures on "bridges" in the unit disk, and we study some of its properties. Section 3 is devoted to the proof of the "universality" result for measures in this class. In Sect. 4, we focus on the corresponding measures on bridges in annuli and their corresponding exponents. In Sect. 5, we discuss the two related models (planar self-avoiding walks and critical percolation) from a nonrigorous perspective. 


\section{Conformally invariant measures}

\subsection{Some preliminaries}

Let $D$ be the open unit disc with boundary $\partial D$. We let $\mathcal{A}=\left(A_{1}, A_{2}\right)$ denote a pair of disjoint closed arcs of $\partial D$, i.e.

$$
\begin{gathered}
A_{1}=A\left(\theta_{1}, \theta_{2}\right)=\left\{e^{i \theta}: \theta \in\left[\theta_{1}, \theta_{2}\right]\right\}, \\
A_{2}=A\left(\theta_{3}, \theta_{4}\right),
\end{gathered}
$$

with $\theta_{1}<\theta_{2}<\theta_{3}<\theta_{4}<\theta_{1}+2 \pi$. Given $A_{1}, A_{2}$, we define

$$
A_{4}=A\left(\theta_{2}, \theta_{3}\right) \text { and } A_{3}=A\left(\theta_{4}, \theta_{1}+2 \pi\right)
$$

so that $A_{1}, A_{4}, A_{2}$ and $A_{3}$ are ordered counterclockwise on the unit circle.

Suppose $D^{\prime} \subset D$ is simply connected and

$$
A_{1} \cup A_{2} \subset \partial D^{\prime} .
$$

By the Riemann mapping theorem, there is a unique

$$
L=L\left(A_{1}, A_{2} ; D^{\prime}\right)
$$

such that there is a conformal map

$$
\Psi: D^{\prime} \rightarrow\{x+i y: 0<x<L, 0<y<\pi\},
$$

with

$$
\begin{gathered}
\Psi\left(A_{1}\right)=\{i y: 0 \leq y \leq \pi\}, \\
\Psi\left(A_{2}\right)=\{L+i y: 0 \leq y \leq \pi\} .
\end{gathered}
$$

We will call $L$ the extremal distance between $A_{1}$ and $A_{2}$ in $D^{\prime}$. Our definition is actually $\pi$ times the usual definition of extremal distance as in, e.g., [1].

For all $\delta \in(0, \pi / 2)$, define

$$
\mathcal{V}_{\delta}=\left(V_{1, \delta}, V_{2, \delta}\right)
$$

where

$$
\begin{aligned}
& V_{1, \delta}=A(\pi-\delta, \pi+\delta), \\
& V_{2, \delta}=A(-\delta, \delta) .
\end{aligned}
$$

There exists a unique $\delta=\delta\left(A_{1}, A_{2} ; D^{\prime}\right)$ such that there is a conformal transformation $\Phi$ mapping $D^{\prime}$ onto the unit disc $D$ in such a way that

$$
\Phi\left(A_{1}\right)=V_{1, \delta} \text { and } \Phi\left(A_{2}\right)=V_{2, \delta} .
$$


If we write

$$
L(\delta)=L\left(V_{1, \delta}, V_{2, \delta} ; D\right),
$$

then $\delta$ is given by

$$
L\left(A_{1}, A_{2} ; D^{\prime}\right)=L(\delta) .
$$

It is not difficult to show that as $\delta \rightarrow 0+$,

$$
L(\delta)=2 \log (1 / \delta)+O(1)
$$

so that

$$
\delta \asymp e^{-L(\delta) / 2}, \quad \delta \rightarrow 0+.
$$

(We write $\asymp$ to means both sides are comparable, i.e., each side is bounded by a constant times the other side.)

\subsection{Bridges}

Let $\mathcal{C}$ denote the set of all connected compact subsets $D^{o}$ of the closed unit disc $\bar{D}$, such that $\bar{D} \backslash D^{o}$ consists of two disjoint connected components that intersect the unit circle. In particular, $\partial D^{o} \cap \partial D$ consists of two disjoint arcs (single points are considered arcs). We call $\mathcal{C}$ the set of bridges in the unit disc.

Suppose that $D^{o} \in \mathcal{C}$, that $d_{1}, d_{2}$ are the two connected components of $\partial D^{o} \cap \partial D$, and that $D_{1}$ and $D_{2}$ are the two connected components of $D \backslash D^{o}$. We label $d_{1}, D_{2}, d_{2}, D_{1}$ in such a way that $d_{1}, \partial D_{2} \cap \partial D, d_{2}, \partial D_{1} \cap \partial D$ are ordered counterclockwise around $\partial D$. There are two possibilities depending on which of the two boundary arcs we call $d_{1}$. We therefore define the set $\mathcal{C}^{o}$ of pairs $\left(D^{o}, d_{1}\right)$ (where $d_{1}$ is one of the two connected components of $\partial D^{o} \cap \partial D$ and $D^{o} \in \mathcal{C}$ ). We call $\mathcal{C}_{0}$ the set of oriented bridges in the unit disc. When $\left(D^{o}, d_{1}\right) \in \mathcal{C}^{o}$, we write

$$
d_{l}=d_{1}, D^{-}=D_{2}, d_{r}=d_{2}, D^{+}=D_{1}
$$

( $l$ and $r$ stand for left and right, + and - for above and below). By a slight abuse of notation, we will often identify $D^{o}$ with $\left(D^{o}, d_{1}\right)$. Note that an element of $\mathcal{C}^{o}$ can be specified either by giving $\left(D^{o}, d_{1}\right)$ or by giving $\left(D^{+}, D^{-}\right)$.

Since we will be interested in intersections between bridges, we endow the set $\mathcal{C}$ with the $\sigma$-field generated by the $\pi$-system of all sets of the form $\left\{D^{o} \in \mathcal{C}: D^{o} \cap K=\emptyset\right\}$ where $K$ ranges over the set $\mathcal{C}^{*}$ of all finite unions of compact connected subsets of $\bar{D}$ that intersect the unit circle. There are other possible choices but this $\sigma$-field will be sufficient for our purposes. 


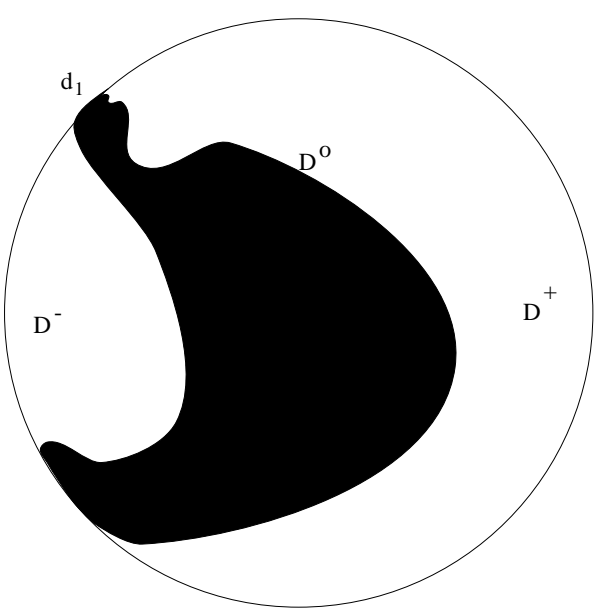

Fig. 1. A bridge

Suppose now that $\mathcal{A}=\left(A_{1}, A_{2}\right)$ is a pair of disjoint arcs as before. Define

$$
\mathcal{C}(\mathcal{A})=\mathcal{C}\left(A_{1}, A_{2}\right)=\left\{\left(D^{o}, d_{l}\right) \in \mathcal{C}^{o}, d_{l} \subset A_{1} \text { and } d_{r} \subset A_{2}\right\} .
$$

This is the set of oriented bridges from $A_{1}$ to $A_{2}$ in $D$. Similarly, if $D^{\prime} \subset D$ with $A_{1} \cup A_{2} \subset \partial D^{\prime}$, we define the set of oriented bridges from $A_{1}$ to $A_{2}$ in $D^{\prime}$ :

$$
\mathcal{C}\left(\mathcal{A} ; D^{\prime}\right)=\mathcal{C}\left(A_{1}, A_{2} ; D^{\prime}\right)=\left\{\left(D^{o}, d_{l}\right) \in \mathcal{C}(\mathcal{A}), D^{o} \cap D \subset D^{\prime}\right\} .
$$

We will often use the set

$$
\mathcal{C}_{\delta}=\mathcal{C}\left(\mathcal{V}_{\delta}\right)=\mathcal{C}\left(V_{1, \delta}, V_{2, \delta}\right)
$$

of oriented bridges from $V_{1, \delta}$ to $V_{2, \delta}$ in $D$.

\subsection{Completely conformal invariant measures}

We are now ready to define our class of measures. We say that the measure $\mu$ on $\mathcal{C}^{o}$ is completely conformally invariant (in short: $\mathrm{CCI}$ ) if it satisfies the following two conditions.

- There exists an $\alpha=\alpha(\mu)>0$ such that as $\delta \rightarrow 0+$,

$$
\mu\left[\mathcal{C}_{\delta}\right] \approx \delta^{2 \alpha}
$$

We call $\alpha$ the crossing exponent of the measure $\mu$. 
- Suppose $D^{\prime}$ is any simply connected subset of $D, \mathcal{A}$ any disjoint pair of arcs on $\partial D \cap \partial D^{\prime}$, and $\Phi$ the corresponding conformal map as before. Let $\mu_{\mathcal{A}, D^{\prime}}$ be the restriction of $\mu$ to the set $\mathcal{C}\left(\mathcal{A} ; D^{\prime}\right)$. The conformal transformation $\Phi$ gives the image measure $\Phi \circ \mu_{\mathcal{A}, D^{\prime}}(\cdot)=\mu_{\mathcal{A}, D^{\prime}}\left(\Phi^{-1}(\cdot)\right)$ on the set $\mathcal{C}(\Phi(\mathcal{A}))=\mathcal{C}\left(\left(\Phi\left(A_{1}\right), \Phi\left(A_{2}\right)\right)\right)$. Then the assumption is

$$
\Phi \circ \mu_{\mathcal{A}, D^{\prime}}=\mu_{\Phi(\mathcal{A}), D}
$$

(for any $\mathcal{A}$ and $D^{\prime}$ ).

The second condition is the most important one; we call it the "conformal and restriction invariance property". Note that when $D^{\prime}=D$, and when $\Phi$ is a Möbius transformation, it implies a usual "invariance under Möbius transformations property" (i.e., if $\Phi$ is a Möbius transformation then $\Phi \circ \mu=\mu$ ). This new property is much stronger.

The crossing exponent measures in some sense how difficult it is to have bridges with "narrow entrance gates".

We now list some general consequences of this definition.

First, note that (3) ensures that, there exists $\delta_{0}>0$ such that $0<$ $\mu\left[\mathcal{C}_{\delta_{0}}\right]<\infty$ so that the mass of bridges with small entrance gates is finite. On the other hand, $\mu$ is necessarily an infinite measure. Take $\delta=\delta_{0}$. It is easy to construct a family of disjoint $\operatorname{arcs}\left(A_{n}, n \geq 0\right)$ such that for any $n$,

$$
L\left(V_{1, \delta}, A_{n} ; D\right)=L\left(V_{1, \delta}, V_{2, \delta} ; D\right)
$$

and therefore, using (4) and the fact that the $A_{n}$ 's are disjoint,

$$
\mu[\mathcal{C}] \geq \sum_{n \geq 0} \mu\left[\mathcal{C}\left(V_{1, \delta}, A_{n}\right)\right]=\sum_{n \geq 0} \mu\left[\mathcal{C}_{\delta}\right]=+\infty .
$$

This shows that it is necessary to consider infinite measures in order to get a conformal invariance property. Note that we used only the invariance under Möbius transformations here.

Suppose that $\Omega$ is a simply connected domain that can be mapped conformally onto the unit disc $D$ via a conformal map $\Psi$. Suppose that $\mathcal{A}$ is a pair of disjoint arcs on $\partial D$ as before and define $U_{1}=\Psi^{-1}\left(A_{1}\right)$ and $U_{2}=\Psi^{-1}\left(A_{2}\right)$ (viewed as sets of prime ends, see [31] for more details). Then, the preimage of the measure $\mu$ on $\mathcal{C}$ is a measure on "bridges" in the domain $\Omega$. This measure inherits the conformal invariance properties from $\mu$, and the crossing exponent measures the asymptotic decay of the mass of the set of "bridges" that connect $U_{1}$ to $U_{2}$ in the limit when the extremal distance $L\left(U_{1}, U_{2} ; \Omega\right)$ tends to infinity. Hence, $\mu$ in fact represents a whole class of measures on "bridges" in (almost) any simply connected domain in the plane. A particularly important example is the $L \times \pi$ rectangle described in (1) with $U_{1}, U_{2}$ being the vertical boundaries. In this case the 
rectangle is conformally equivalent to the circle, and $U_{1}, U_{2}$ can be mapped conformally onto the arcs $V_{1, \delta}, V_{2, \delta}$ for some $\delta=\delta(L)$. Note that

$$
\delta(L) \asymp e^{-L / 2}, \quad L \rightarrow \infty .
$$

In particular, the crossing exponent $\alpha$ can be interpreted as follows: The mass of the set bridges between $U_{1}$ and $U_{2}$ in the $L \times \pi$ rectangle decays roughly like $\exp (-\alpha L)$ when $L \rightarrow \infty$.

Proposition 1. For any CCI measure $\mu$, the crossing exponent $\alpha(\mu)$ is greater or equal to 1 .

Proof. Define the upper and lower half-planes

$$
H^{+}=\{z \in \mathbb{C}: \Im(z)>0\} \text { and } H^{-}=\{z \in \mathbb{C}: \Im(z)<0\} .
$$

For any $\epsilon \in\{+,-\}$ and $\kappa \in\{1,2\}$ and $\delta>0$, put

$$
V_{\kappa, \delta}^{\epsilon}=V_{\kappa, \delta} \cap H^{\epsilon} .
$$

It is very easy to check that for any $\delta<\pi / 4$, for any $\epsilon_{1}, \epsilon_{2} \in\{-,+\}$,

$$
L\left(V_{1, \delta}^{\epsilon_{1}}, V_{2, \delta}^{\epsilon_{2}} ; D\right) \leq L\left(V_{1, \delta / 2}, V_{2, \delta / 2} ; D\right)=L(\delta / 2) .
$$

Define

$$
f(\delta)=\mu\left[\mathcal{C}_{\delta}\right] .
$$

Clearly $f$ is a non-decreasing function of $\delta$ and

$$
f(\delta) \geq \sum_{\epsilon_{1}, \epsilon_{2} \in\{+,-\}} \mu\left[\mathcal{C}\left(V_{1, \delta}^{\epsilon_{1}}, V_{2, \delta}^{\epsilon_{2}}\right)\right] .
$$

Then, conformal invariance and non-decrease of $f$ shows that

$$
f(\delta) \geq \sum_{\epsilon_{1}, \epsilon_{2} \in\{+,-\}} f(\delta / 2)=4 f(\delta / 2) .
$$

Hence, as $f$ is non-decreasing and $f\left(\delta_{0}\right)<\infty$ we conclude that there exists $c<\infty$ such that for any $\delta<\delta_{0}$,

$$
f(\delta) \leq c \delta^{2}
$$

so that $\alpha(\mu) \geq 1$ and this concludes the proof of the proposition. We shall see that $\alpha(\mu)=1$ in the case of the Brownian excursion measure. 


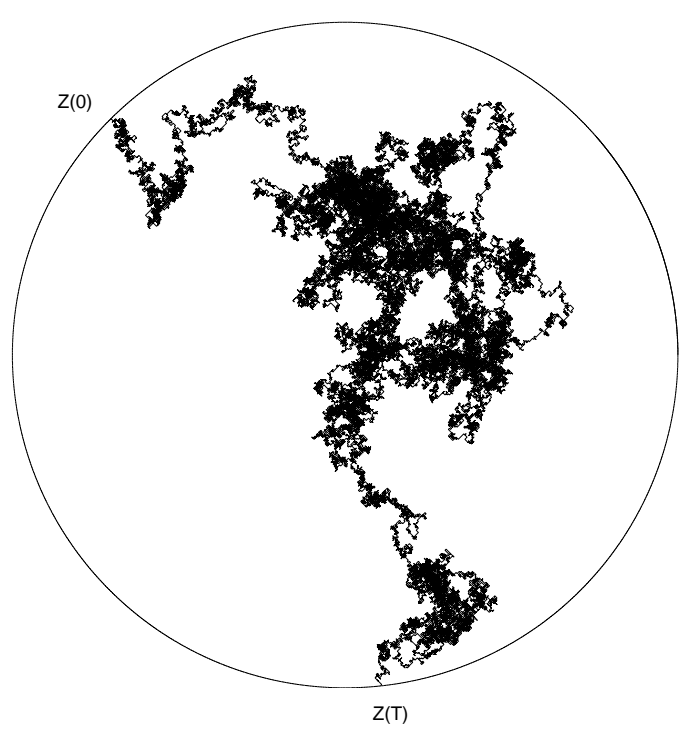

Fig. 2. A Brownian excursion

\subsection{Brownian excursion measure}

As a first example, we construct the Brownian excursion measure in $D$, which was implicitly used in [27].

Lemma 2. There exists a CCI measure v corresponding to hulls of Brownian excursions in $D$, with $\alpha(v)=1$.

We will start by defining the excursion measure as a measure on paths in the half-plane $H=\{z: \Im(z)>0\}$ and then use conformal invariance to extend it to other simply connected domains. An excursion on a domain $\Omega$ is a continuous path $Z:[0, T] \rightarrow \bar{\Omega}$ for some positive $T$, such that $Z(0), Z(T) \in \partial \Omega$ and $Z(0, T) \subset \Omega$. Throughout this subsection, we will implicitly use the topology (and weak convergence) associated with the distance between continuous paths $Z:[0, T] \rightarrow \mathbb{C}$ and $Z^{\prime}:\left[0, T^{\prime}\right] \rightarrow \mathbb{C}$ defined by $d\left(Z, Z^{\prime}\right)=\inf _{\phi} \sup _{t \in[0, T]}\left|Z(T)-Z^{\prime}(\phi(t))\right|$ where $\phi$ ranges over all increasing bijections between $[0, T]$ and $\left[0, T^{\prime}\right]$. In particular, we identify $Z$ and $Z^{\prime}$ when $Z^{\prime}$ can be obtained by increasing reparametrization of $Z$.

Itô's one-dimensional excursion measure is an infinite measure on paths $Y:[0, T] \rightarrow[0, \infty)$ with $Y_{0}, Y_{T}=0$ and $Y_{t}>0$ for $0<t<T$. The measure can be constructed as

$$
\lim _{\epsilon \rightarrow 0+} \epsilon^{-1} \mu_{\epsilon}
$$

where $\mu_{\epsilon}$ is the probability measure given by starting a Brownian motion at $\epsilon$ and killing it when it reaches the origin (see e.g. [32]). The (twodimensional) excursion measure in the half-plane $H$ starting at $x \in \mathbb{R}$ is 
the measure $v_{H, x}$ given by $X_{t}+i Y_{t}$ where $Y_{t}$ is an excursion and $X_{t}$ is an independent Brownian motion starting at $x$ and stopped at the same time as $Y$. This is an infinite measure. It can also be thought of as a consistent family of finite measures $\left\{v_{H, x, A}\right\}$ where $A$ is a closed subset of $\mathbb{R}$ not containing $x$. The measure $v_{H, x, A}$ denotes the measure $v_{H, x}$ restricted to paths that terminate in $A$. If $z_{n} \in H$ with $z_{n} \rightarrow x$, and $v_{H, z_{n}, A}$ denotes the measure on Brownian paths started at $z_{n}$ killed upon reaching the real axis, restricted to paths that hit $\mathbb{R}$ at $A$, then it is easy to see that

$$
\Im\left(z_{n}\right)^{-1} v_{H, z_{n}, A} \longrightarrow v_{H, x, A} .
$$

Suppose $\Phi: H \rightarrow H$ is a Möbius transformation with $\Phi(x) \in \mathbb{R}$. Then for each closed $A \subset \overline{\mathbb{R}}$ with $x \notin A$, using conformal invariance of Brownian motion (and the fact that $\Phi$ is continuous on the set of excursions starting at $x$ and ending in $A$ ),

$$
\begin{aligned}
\Phi \circ v_{H, x, A} & =\lim _{\epsilon \rightarrow 0+} \Phi\left(\epsilon^{-1} v_{H, x+i \epsilon, A}\right) \\
& =\lim _{\epsilon \rightarrow 0+} \epsilon^{-1} v_{H, \Phi(x+i \epsilon), \Phi(A)} \\
& =\lim _{\epsilon \rightarrow 0+} \epsilon^{-1} v_{H, \Phi(x)+i\left|\Phi^{\prime}(x)\right| \epsilon+o(\epsilon), \Phi(A)} \\
& =\left|\Phi^{\prime}(x)\right| v_{H, \Phi(x), \Phi(A)} .
\end{aligned}
$$

If $A_{1}, A_{2}$ are disjoint closed subsets of $\overline{\mathbb{R}}$, we define

$$
v_{H, A_{1}, A_{2}}=\int_{A_{1}} v_{H, x, A_{2}} d x .
$$

If $\Phi$ is a Möbius transformation from $H$ onto $H$,

$$
\begin{aligned}
\Phi \circ v_{H, A_{1}, A_{2}} & =\int_{A_{1}} \Phi\left(v_{H, x, A_{2}}\right) d x \\
& =\int_{A_{1}}\left|\Phi^{\prime}(x)\right| v_{H, \Phi(x), \Phi\left(A_{2}\right)} d x \\
& =\int_{\Phi\left(A_{1}\right)} v_{H, y, \Phi\left(A_{2}\right)} d y=v_{H, \Phi\left(A_{1}\right), \Phi\left(A_{2}\right)} .
\end{aligned}
$$

Note that the scaling factor $\left|\Phi^{\prime}(x)\right|$ is balanced by the change of variable in the integration over the starting points in $A_{1}$. We now define the excursion measure $v_{H}$ to be the consistent set of measures $\left\{v_{H, A_{1}, A_{2}}\right\}$, or equivalently,

$$
v=\int_{\mathbb{R}} v_{H, x} d x .
$$

The measure $v_{H}$ is invariant under Möbius transformations of $H$ by (6). 
Suppose that $\Phi$ is a conformal mapping from $H$ onto a simply connected domain $\Omega$, such that $\Phi^{\prime}$ can be extended continuously to $x \in \mathbb{R}$. Let $A$ be a closed interval in $\mathbb{R}$ not containing $x$ and let $\Phi(x)=\tilde{x}, \Phi(A)=\tilde{A}$. Then, we define the measure $v_{\Omega, \tilde{x}, \tilde{A}}$ on excursions in $\Omega$, started from $\tilde{x}$ by

$$
v_{\Omega, \tilde{x}, \tilde{A}}=\left|\Phi^{\prime}(x)\right| \Phi \circ v_{H, x, A} .
$$

By (5), this definition is independent of the choice of $x, A$ and $\Phi$. Similarly, if $A_{1}, A_{2}$ are disjoint closed subsets of $\overline{\mathbb{R}}, \Phi\left(A_{1}\right)=\tilde{A}_{1}, \Phi\left(A_{2}\right)=\tilde{A}_{2}$, and $\Phi^{\prime}$ can be extended continuously to $A_{1}$, we define

$$
\begin{array}{r}
v_{\Omega, \tilde{A}_{1}, \tilde{A}_{2}}=\int_{A_{1}}\left|\Phi^{\prime}(x)\right| \Phi \circ v_{H, x, A_{2}} d x \\
=\int_{\tilde{A}_{1}} v_{\Omega, y, \tilde{A}_{2}} d \lambda(y) .
\end{array}
$$

Here $\lambda(\cdot)$ denotes Lebesgue measure on $\tilde{A}_{1}$. The measure $v_{\Omega}$ is defined similarly.

Suppose now $\Omega$ is a simply connected subset of the upper half-plane whose boundary contains a real neighborhood of $x$ and the closed set $\tilde{A} \subset \overline{\mathbb{R}}$ with $x \notin \tilde{A}$, and let $\Phi$ be a conformal mapping from $H$ onto $\Omega$ with $\Phi(x)=x$ and $\Phi^{\prime}(x)=1$. Let $A=\Phi^{-1}(\tilde{A})$. Conformal invariance of planar Brownian motion and the fact that $\Phi(x+i \epsilon)=x+i \epsilon+o(\epsilon)$ show easily that $v_{\Omega, x, \tilde{A}}$ is the limit when $\epsilon \rightarrow 0$ of $\epsilon^{-1} \tilde{P}^{x+i \epsilon}$ where $\tilde{P}^{x+i \epsilon}$ is the law of Brownian motion $B$ started from $x+i \epsilon$, killed at its first exit $Z(T)$ of $\Omega$, restricted to the event $Z(T) \in \tilde{A}$. This shows readily that the measure $v_{\Omega, x, \tilde{A}}$ can be obtained by restricting $v_{H, x}$ to the set of excursions with $Z(0, T) \subset \Omega$ and $Z(T) \in \tilde{A}$ :

$$
v_{\Omega, x, A}=v_{H, x, A} 1_{\{Z(0, T) \subset \Omega\}} .
$$

Similarly, if $\partial \Omega$ contains disjoint closed subsets $A_{1}, A_{2}$ of $\overline{\mathbb{R}}$,

$$
v_{\Omega, A_{1}, A_{2}}=v_{H, A_{1}, A_{2}} 1_{\{Z(0, T) \subset \Omega\}} .
$$

For $\Omega=D$, we obtain the excursion measure on the disk $v_{D}$. It is easy to see that this is the same as the limit of $2 \pi \epsilon^{-1}$ times the probability measure on Brownian paths started uniformly on the circle of radius $1-\epsilon$, killed when they reach the unit circle. Suppose now that (as in the definition of the CCI property) $D^{\prime}$ is a simply connected subset of the unit disc $D$, that the two arcs $A_{1}$ and $A_{2}$ are disjoint subsets of $\partial D^{\prime} \cap \partial D$, and that $\Phi$ is a conformal mapping from $D^{\prime}$ onto $D$ that maps $A_{1}$ onto $V_{1, \delta}$ and $A_{2}$ onto $V_{2, \delta}$ (for well-chosen $\delta$ ). By mapping $D$ and $D^{\prime}$ back onto the upper half- 
plane, and using (7), we get that the measure $v_{D}$ restricted to the excursions such that $\left\{Z(0) \in A_{1}, Z(T) \in A_{2}, Z(0, T) \subset D^{\prime}\right\}$, is the conformal image of the measure $v_{D}$ restricted to $\left\{Z(0) \in V_{1, \delta}, Z(T) \in V_{2, \delta}\right\}$. Also, it is straightforward to check directly that

$$
v_{D}\left(\left\{Z(0) \in V_{1, \delta}, Z(T) \in V_{2, \delta}\right\}\right) \approx \delta^{2}, \quad \text { when } \delta \rightarrow 0+.
$$

To each excursion $Z$ in the disk such that $Z(0) \neq Z(T)$, one can the associate an oriented bridge as follows. Consider the complement $D^{o}$ (in $\mathbb{C}$ ) of the unbounded connected component of $\mathbb{C} \backslash Z[0, T]$. In other words, $D^{o}$ is obtained by filling in the holes of the trace of $Z$. Then, $\left(D^{o},\{Z(0)\}\right)$ is an oriented bridge. It is easy to check that the mapping $Z \mapsto D^{o}$ is measurable, so that any measure on excursions defines an image measure on oriented bridges. We use $v$ to denote to image of $v_{D}$. The properties of $v_{D}$ that we just derived show immediately that $v$ is a CCI measure with $\alpha(v)=1$

\subsection{Unions of bridges}

Let $p$ be a positive integer and suppose that $\mu_{1}, \ldots, \mu_{p}$ are CCI measures on $\mathcal{C}^{o}$. Define independently (i.e. with respect to the product measure)

$$
\left(D_{1}^{+}, D_{1}^{o}, D_{1}^{-}\right),\left(D_{2}^{+}, D_{2}^{o}, D_{2}^{-}\right), \ldots,\left(D_{p}^{+}, D_{p}^{o}, D_{p}^{-}\right)
$$

using the measures $\mu_{1}, \ldots, \mu_{p}$ respectively. Then define

$$
\begin{aligned}
& D^{+}=D_{1}^{+} \cap D_{2}^{+} \cap \cdots \cap D_{p}^{+} \\
& D^{-}=D_{1}^{-} \cap D_{2}^{-} \cap \cdots \cap D_{p}^{-} .
\end{aligned}
$$

Let $\mu=\cup\left(\mu_{1}, \cdots, \mu_{p}\right)$ be the measure corresponding to $\left(D^{+}, D^{-}\right)$(restricted to the event $\left\{D^{+} \neq \varnothing\right.$ and $\left.\left.D^{-} \neq \varnothing\right\}\right)$. It is then easy to check that $\mu$ is also a CCI measure. Note that

$$
\alpha(\mu)=\alpha\left(\mu_{1}\right)+\alpha\left(\mu_{2}\right)+\cdots+\alpha\left(\mu_{p}\right) .
$$

This last procedure with

$$
\mu_{1}=\mu_{2}=\cdots=\mu_{p}=v
$$

defines a measure that we will denote by $\nu^{\cup p}$. This is the measure corresponding to the "unions of $p$ independent Brownian excursions" in $D$. 


\section{Intersection exponents}

\subsection{Intersection exponents and crossing exponent}

Suppose now that $p>1$ and that $\mu_{1}, \ldots, \mu_{p}$ are CCI measures. Define

$$
\left(D_{1}^{+}, D_{1}^{o}, D_{1}^{-}\right), \ldots,\left(D_{p}^{+}, D_{p}^{o}, D_{p}^{-}\right)
$$

independently (i.e. according to the product measure) using the measures $\mu_{1}, \ldots, \mu_{p}$.

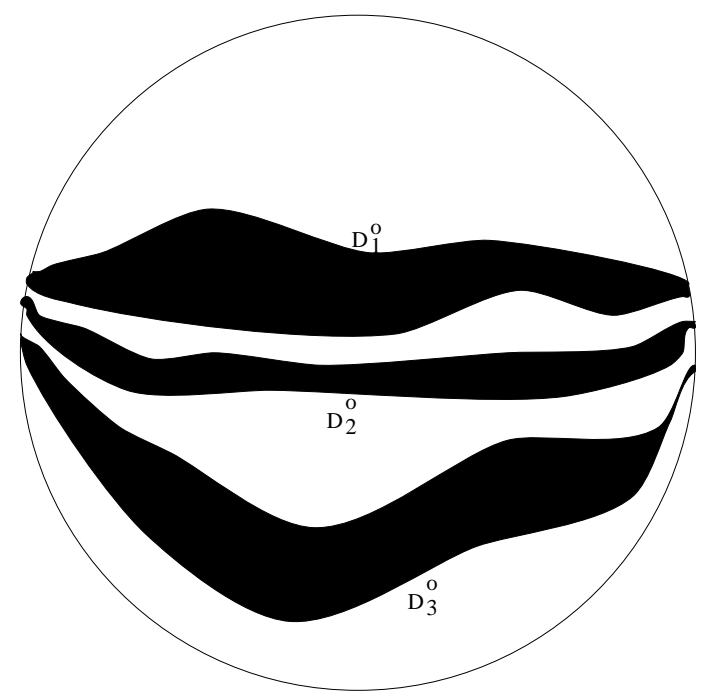

Fig. 3. Three ordered bridges

We say that the $p$ (oriented) bridges $D_{1}^{o}, \ldots, D_{p}^{o}$ are ordered if

$$
D_{j}^{o} \cap D \subset D_{j+1}^{-}, \quad j=1, \ldots, p-1 .
$$

Let $\mathcal{O}=\mathcal{O}(p)$ denote this event. Note that this event is the same as

$$
D_{j}^{o} \cap D \subset D_{j-1}^{+}, \quad j=2, \ldots, p .
$$

The intersection exponent $\alpha=\alpha\left(\mu_{1}, \ldots, \mu_{p}\right)$ is defined by

$$
\mu_{1} \otimes \cdots \otimes \mu_{p}\left(\left(\mathcal{C}_{\delta}\right)^{p} \cap \mathcal{O}\right) \approx \delta^{2 \alpha}, \quad \delta \rightarrow 0+,
$$

in the case where such an $\alpha$ exists. In other words, $\delta^{2 \alpha}$ is the approximate measure of the set of bridges such that for all $j^{\prime}<j$ in $\{1, \cdots, p\}, D_{j^{\prime}}^{o}$ and $D_{j}^{o}$ are in $\mathcal{C}_{\delta}$ and

$$
D_{j^{\prime}}^{o} \text { stays "strictly below" } D_{j}^{o} \text {. }
$$


Note that the intersection exponent $\alpha$ can be viewed as the crossing exponent of another single CCI measure (and this justifies the fact that we use the same notation). If $\mathcal{O}$ is satisfied, define

$$
\begin{aligned}
& D^{+}=D_{p}^{+}=D_{1}^{+} \cap \cdots \cap D_{p}^{+}, \\
& D^{-}=D_{1}^{-}=D_{p}^{-} \cap \cdots \cap D_{p}^{-}
\end{aligned}
$$

and the corresponding $D^{o}$. The measure $\mu_{1} \otimes \cdots \otimes \mu_{p}$ restricted to the event $\mathcal{O}$ thus defines a measure $\mu=\Xi\left(\mu_{1}, \cdots, \mu_{p}\right)$, and it is easy to check that this is a CCI measure on $\mathcal{C}^{o}$ with crossing exponent $\alpha(\mu)=\alpha\left(\mu_{1}, \cdots, \mu_{p}\right)$.

\subsection{Some results for Brownian intersection exponents}

We now review some of the definitions and results derived in [27].

- In the case $\mu_{1}=\cdots=\mu_{p}=v$, the exponent $\alpha\left(\mu_{1}, \ldots, \mu_{p}\right)$ is known to exist and it is exactly the Brownian intersection exponent $\tilde{\xi}\left(1^{\otimes p}\right)$ for $p$ Brownian paths in a half-plane as defined in [27].

- Suppose that $n_{1}, \cdots, n_{p}$ are positive integers. Then the exponent

$$
\alpha\left(\nu^{\cup n_{1}}, \ldots, \nu^{\cup n_{p}}\right)
$$

exists. This exponent is called the "intersection exponent between packs of Brownian motions in the half-plane" and is denoted by

$$
\tilde{\xi}\left(n_{1}, \ldots, n_{p}\right) .
$$

- There exists a unique natural (see [27]) extension of

$$
\tilde{\xi}\left(\lambda_{1}, \ldots, \lambda_{p}\right)
$$

for any nonnegative reals $\lambda_{1}, \ldots, \lambda_{p}$ in such a way that for any $j \in$ $\{1, \ldots, p-1\}$,

$$
\tilde{\xi}\left(\lambda_{1}, \ldots, \lambda_{j}, \tilde{\xi}\left(\lambda_{j+1}, \ldots, \lambda_{p}\right)\right)=\tilde{\xi}\left(\lambda_{1}, \ldots, \lambda_{p}\right)
$$

(we called this relation the "cascade relation"), and for any permutation $\sigma$ of $\{1, \ldots, p\}$,

$$
\tilde{\xi}\left(\lambda_{\sigma(1)}, \ldots, \lambda_{\sigma(p)}\right)=\tilde{\xi}\left(\lambda_{1}, \ldots, \lambda_{p}\right) .
$$

Also,

$$
\tilde{\xi}\left(\lambda_{1}, \ldots, \lambda_{p}, 0\right)=\tilde{\xi}\left(\lambda_{1}, \ldots, \lambda_{p}\right) .
$$


$\tilde{\xi}\left(\lambda_{1}, \cdots, \lambda_{p}\right)$ can be interpreted as the intersection exponent for packs of Brownian motions in a half-space such that each pack is respectively the union of $\lambda_{1}, \cdots, \lambda_{p}$ Brownian paths (even though this is not realizable when the $\lambda$ 's are not integers). The values of all exponents $\tilde{\xi}$ are determined by the function

$$
\left(\lambda, \lambda^{\prime}\right) \longmapsto \tilde{\xi}\left(\lambda, 1, \lambda^{\prime}\right) .
$$

Note also that $\lambda \mapsto \tilde{\xi}(1, \lambda)$ is bijective on $[0, \infty)$ (see [27] for all these results).

Let us now say a few words about how $\tilde{\xi}\left(\lambda, 1, \lambda^{\prime}\right)$ can be defined in the present context. In order to clarify the notation of the subsequent proofs, we will use the following notation: Suppose that $W=W^{o}$ is defined under the CCI measure $v$. In other words, $W$ is the hull of a Brownian excursion in the unit disc (with specified initial point). We will write $W^{+}=D^{+}(W), W^{-}=$ $D^{-}(W)$ for the corresponding sets. If $W \in \mathcal{C}_{\delta}$, define for $\epsilon \in\{+,-\}$,

$$
\begin{aligned}
V_{1, \delta}^{\epsilon}(W) & =V_{1, \delta} \cap \partial W^{\epsilon} \\
V_{2, \delta}^{\epsilon}(W) & =V_{2, \delta} \cap \partial W^{\epsilon} \\
L_{\delta}^{\epsilon}(W) & =L\left(V_{1, \delta}^{\epsilon}(W), V_{2, \delta}^{\epsilon}(W) ; W^{\epsilon}\right) .
\end{aligned}
$$

Then, the intersection exponent $\tilde{\xi}\left(\lambda, 1, \lambda^{\prime}\right)$ is characterized by the relation:

$$
\begin{aligned}
& v\left(1_{\left\{W \in \mathcal{C}_{\delta}\right\}} \exp \left\{-\lambda L_{\delta}^{+}(W)-\lambda^{\prime} L_{\delta}^{-}(W)\right\}\right) \\
& \quad \approx \exp \left\{-\tilde{\xi}\left(\lambda, 1, \lambda^{\prime}\right) L(\delta)\right\}, \quad \delta \rightarrow 0+.
\end{aligned}
$$

It can be easily shown using Schwarz's inequality immediately (see [27]) that the mapping $\left(\lambda, \lambda^{\prime}\right) \mapsto \tilde{\xi}\left(\lambda, 1, \lambda^{\prime}\right)$ is concave. In [25], the following result is proved.

Proposition 3. Let $\lambda^{\prime} \geq 0$ and let $g(\lambda)=\tilde{\xi}\left(\lambda, 1, \lambda^{\prime}\right)$. Then $g(\lambda)$ is $C^{2}$ for $\lambda>0$ with $g^{\prime \prime}(\lambda)<0$.

Let $\lambda^{\prime}=0$, and let

$$
a(\lambda)=\frac{\partial}{\partial \lambda} \tilde{\xi}(\lambda, 1)
$$

Then the strict concavity of $g$ can be used to show that for every $\epsilon>0$,

$$
v\left(1_{\left\{W \in \mathcal{C}_{\delta}\right\}} \exp \left\{-\lambda L_{\delta}^{+}\right\} 1_{\left\{\left|L_{\delta}^{+}(W)-a(\lambda) L(\delta)\right| \leq \epsilon L(\delta)\right\}}\right) \approx \exp \{-\tilde{\xi}(\lambda, 1) L(\delta)\} .
$$

The following lemma is then a simple corollary of Proposition 3. 
Lemma 4. Suppose $\lambda>0$ is fixed and that $\Psi: \mathbb{R}_{+} \rightarrow \mathbb{R}_{+}$is a nonincreasing function such that when $\delta \rightarrow 0+$,

$$
v\left(1_{\left\{W \in \mathcal{C}_{\delta}\right\}} \Psi\left(L_{\delta}^{+}(W)\right)\right) \approx \exp \{-\tilde{\xi}(\lambda, 1) L(\delta)\}
$$

then

$$
\Psi(l) \approx \exp (-\lambda l), l \rightarrow \infty .
$$

Let us now conclude this section with the following observation: Define $\mu=\Xi(\nu, v)$ as in the previous section with the corresponding sets

$$
D^{+}=D_{2}^{+}=D_{1}^{+} \cap D_{2}^{+} \text {and } D^{-}=D_{1}^{-}=D_{1}^{-} \cap D_{2}^{-}
$$

(when the second Brownian excursion is "above" the first one). Now, if this event holds, define

$$
D^{m}=D_{1}^{+} \cap D_{2}^{-} .
$$

This is the set "between" the two excursions. Call $\partial_{1}^{m}, \partial_{2}^{m}$ the two connected components of $D^{m} \cap \partial D$, and

$$
L^{m}=L\left(\partial_{1}^{m}, \partial_{2}^{m} ; D^{m}\right) .
$$

Then, define $\mu_{1, \lambda, 1}$ by

$$
d \mu_{1, \lambda, 1}=\exp \left(-\lambda L^{m}\right) d \mu .
$$

It is easy to check that $\mu_{1, \lambda, 1}$ satisfies the conformal and restriction invariance property (simply because $L^{m}$ is a conformal invariant) and (see [27]) that it has crossing exponent $\tilde{\xi}(1, \lambda, 1)$. Note that the function $\lambda \mapsto \tilde{\xi}(1, \tilde{\xi}(1, \lambda))$ is a bijection from $\mathbb{R}_{+}$onto $[\tilde{\xi}(1,1), \infty)$, so that:

Proposition 5. For any $\alpha_{0} \geq \tilde{\xi}(1,1)$, there exists a CCI measure $\mu$ with $\alpha(\mu)=\alpha_{0}$.

\subsection{Universality}

Suppose now that $\mu$ is a CCI measure on $\mathcal{C}^{o}$. Recall that this gives a measure on pairs $\left(D^{o}, d_{1}\right)$, or equivalently, on pairs $\left(D^{+}, D^{-}\right)$. If $D^{o} \in \mathcal{C}_{\delta}, \epsilon \in$ $\{+,-\}$, define, as before,

$$
\begin{gathered}
V_{1, \delta}^{\epsilon}=V_{1, \delta}^{\epsilon}\left(D^{o}\right)=V_{1, \delta} \cap \partial D^{\epsilon} \\
V_{2, \delta}^{\epsilon}=V_{2, \delta}^{\epsilon}\left(D^{o}\right)=V_{2, \delta} \cap \partial D^{\epsilon} \\
L_{\delta}^{\epsilon}=L_{\delta}^{\epsilon}\left(D^{o}\right)=L\left(V_{1, \delta}^{\epsilon}, V_{2, \delta}^{\epsilon} ; D^{\epsilon}\right) .
\end{gathered}
$$

Define for all $\lambda>0$ and $\delta \in(0, \pi / 2)$,

$$
\Psi^{\epsilon}(\lambda, L(\delta))=\mu\left(\exp \left\{-\lambda L_{\delta}^{\epsilon}\right\} 1_{\left\{D^{o} \in \mathcal{C}_{\delta}\right\}}\right) .
$$


The generalized exponent $\beta^{\epsilon}(\mu, \lambda)$ is defined by

$$
\Psi^{\epsilon}(\lambda, L(\delta)) \approx \exp \left\{-L(\delta) \beta^{\epsilon}(\mu, \lambda)\right\}, \quad \delta \rightarrow 0+,
$$

in the case where such a $\beta^{\epsilon}(\mu, \lambda)$ exists.

Theorem 6. For any $\lambda>0$, the generalized intersection exponents $\beta^{+}(\mu, \lambda)$ and $\beta^{-}(\mu, \lambda)$ exist. They are equal, and they are also equal to

$$
\beta(\mu, \lambda)=\tilde{\xi}(\alpha(\mu), \lambda) .
$$

Proof. We will show that $\beta^{+}(\mu, \lambda)=\tilde{\xi}(\alpha(\mu), \lambda)$; a similar argument works for $\beta^{-}(\mu, \lambda)$. Let us first introduce some notation. Suppose that $\left(D^{o}, d_{1}\right)$, or equivalently, $\left(D^{+}, D^{-}\right)$, is chosen using the CCI measure $\mu$. If $D^{o} \in \mathcal{C}_{\delta}$, define $L_{\delta}^{+}$as above. Define independently $W=W^{o}$ under the measure $v$. In other words, $W$ is the hull of a Brownian excursion. If $W \in \mathcal{C}_{\delta}$, we define $L_{\delta}^{+}(W), L_{\delta}^{-}(W)$ and the domains $W^{+}, W^{-}$as before.

Suppose now that $W$ is "above" $D^{o}$, i.e., that $W \subset D^{+}$(more precisely, we should say $W \cap D \subset D^{+}$, but for notational ease we will write just $\left.W \subset D^{+}\right)$. Let $\tilde{D}=\tilde{D}\left(D^{o}, W\right)$ be the domain between $D^{o}$ and $W$ :

$$
\tilde{D}=\tilde{D}\left(D^{o}, W\right)=D^{+} \cap W^{-} .
$$

We define the corresponding extremal distance

$$
\tilde{L}_{\delta}=\tilde{L}_{\delta}\left(D^{o}, W\right)=L\left(V_{1, \delta} \cap \partial \tilde{D}, V_{2, \delta} \cap \partial \tilde{D} ; \tilde{D}\right) .
$$

Let $\lambda>0$ be fixed. We are going to study the asymptotic behavior as $\delta \rightarrow 0+$ of $Q(\delta)$ defined by

$$
Q(\delta)=\mu \otimes v\left(1_{\left\{W \in \mathcal{C}_{\delta}, D^{o} \in \mathcal{C}_{\delta}, W \subset D^{+}\right\}} \exp \left\{-\lambda \tilde{L}_{\delta}\right\}\right) .
$$

Suppose that we integrate first with respect to $\nu$. For any fixed $D^{+}$, symmetry of the Brownian excursion measure in $D^{+}$, which can be established by conformal invariance, shows that

$$
v\left(1_{\left\{W \in \mathcal{C}_{\delta}, W \subset D^{+}\right\}} \exp \left\{-\lambda \tilde{L}_{\delta}\right\}\right)=v\left(1_{\left\{W \in \mathcal{C}_{\delta}, W \subset D^{+}\right\}} \exp \left\{-\lambda L_{\delta}^{+}(W)\right\}\right) .
$$

Hence, we can replace $\tilde{L}_{\delta}$ with $L_{\delta}^{+}(W)$ in the definition of $Q(\delta)$. In particular, by changing the order of integration, we see that

$$
Q(\delta)=v\left(1_{\left\{W \in \mathcal{C}_{\delta}\right\}} \exp \left\{-\lambda L_{\delta}^{+}(W)\right\} \mu\left(\left\{D^{o} \in \mathcal{C}_{\delta} \text { and } D^{o} \subset W^{-}\right\}\right)\right) .
$$

But complete conformal invariance of $\mu$ and the definition of $\alpha(\mu)$ show that when $\delta \rightarrow 0+$,

$$
\mu\left(\left\{D^{o} \in \mathcal{C}_{\delta} \text { and } D^{o} \subset W^{-}\right\}\right) \approx \exp \left\{-\alpha(\mu) L_{\delta}^{-}(W)\right\} .
$$


Hence,

$$
Q(\delta) \approx \exp \{-\tilde{\xi}(\lambda, 1, \alpha(\mu)) L(\delta)\}, \quad \delta \rightarrow 0+.
$$

On the other hand, if we use the initial definition of $Q(\delta)$ and the complete conformal invariance of $\mu$, we see that when $\delta \rightarrow 0+$,

$$
\begin{aligned}
Q(\delta) & =v\left(1_{\left\{W \in \mathcal{C}_{\delta}\right\}} \mu\left[1_{\left\{D^{o} \in \mathcal{C}_{\delta}, D^{o} \subset W^{-}\right\}} \exp \left\{-\lambda \tilde{L}_{\delta}\right\}\right]\right) \\
& =v\left(1_{\left\{W \in \mathcal{C}_{\delta}\right\}} \Psi^{+}\left(\lambda, L_{\delta}^{-}(W)\right)\right) .
\end{aligned}
$$

We know from (10) that this quantity is logarithmically asymptotic to

$$
\exp \{-L(\delta) \tilde{\xi}(\lambda, 1, \alpha(\mu))\} .
$$

But (8) and (9) imply that

$$
\tilde{\xi}(\lambda, 1, \alpha(\mu))=\tilde{\xi}(1, \alpha(\mu), \lambda)=\tilde{\xi}(1, \tilde{\xi}(\alpha(\mu), \lambda)) .
$$

Hence we can apply Lemma 4 to deduce that

$$
\Psi^{+}(\lambda, l) \approx \exp \{-l \tilde{\xi}(\alpha(\mu), \lambda)\}, \quad l \rightarrow \infty .
$$

In other words, $\beta^{+}(\mu, \lambda)$ exists and

$$
\beta^{+}(\mu, \lambda)=\tilde{\xi}(\alpha(\mu), \lambda) .
$$

This completes the proof.

Theorem 7. Suppose that $\mu_{1}, \ldots, \mu_{p}$ are CCI measures. Then the intersection exponent $\alpha\left(\mu_{1}, \ldots, \mu_{p}\right)$ exists and

$$
\alpha\left(\mu_{1}, \ldots, \mu_{p}\right)=\tilde{\xi}\left(\alpha\left(\mu_{1}\right), \ldots, \alpha\left(\mu_{p}\right)\right) .
$$

Proof. We proceed by induction over $p$. The case $p=1$ is trivial. Assume that $p \geq 2$, that $\alpha\left(\mu_{2}, \ldots, \mu_{p}\right)$ exists and is equal to $\tilde{\xi}\left(\alpha\left(\mu_{2}\right), \ldots, \alpha\left(\mu_{p}\right)\right)$. Complete conformal invariance of the measures $\mu_{1}, \ldots, \mu_{p}$ shows immediately that $\alpha\left(\mu_{1}, \ldots, \mu_{p}\right)$ exists and that

$$
\alpha\left(\mu_{1}, \ldots, \mu_{p}\right)=\beta\left(\mu_{1}, \alpha\left(\mu_{2}, \ldots, \mu_{p}\right)\right) .
$$

Hence,

$$
\begin{aligned}
\alpha\left(\mu_{1}, \ldots, \mu_{p}\right) & =\tilde{\xi}\left(\alpha\left(\mu_{1}\right), \alpha\left(\mu_{2}, \ldots, \mu_{p}\right)\right) \\
& =\tilde{\xi}\left(\alpha\left(\mu_{1}\right), \tilde{\xi}\left(\alpha\left(\mu_{2}\right), \ldots, \alpha\left(\mu_{p}\right)\right)\right) \\
& =\tilde{\xi}\left(\alpha\left(\mu_{1}\right), \alpha\left(\mu_{2}\right), \ldots, \alpha\left(\mu_{p}\right)\right) .
\end{aligned}
$$

and the theorem is proved. 
In particular, $\alpha\left(\mu_{1}, \ldots, \mu_{p}\right)=\alpha\left(\mu_{\sigma(1)}, \ldots, \mu_{\sigma(p)}\right)$ for any permutation $\sigma$ of $\{1, \cdots, p\}$ as $\tilde{\xi}$ is invariant under permutation. Hence, $\alpha$ is the ("unordered") intersection exponent associated to $\mu_{1}, \cdots, \mu_{p}$ in the following sense:

$$
\begin{aligned}
\mu_{1} \otimes \cdots \otimes \mu_{p}\left(\left\{\forall j \neq j^{\prime}\right.\right. & \left.\left.\in\{1, \ldots, p\}, D_{j}^{o} \cap D_{j^{\prime}}^{o}=\emptyset\right\} \cap \mathcal{C}_{\delta}^{p}\right) \\
& \approx \exp \left(-L(\delta) \alpha\left(\mu_{1}, \ldots, \mu_{p}\right)\right), \quad \delta \rightarrow 0+.
\end{aligned}
$$

Indeed, if the sets $D_{j}^{o}$ are disjoint in $\mathcal{C}_{\delta}$, then for some permutation $\sigma$ of $\{1, \ldots, p\}$, the $p$ bridges $\left(D_{\sigma(1)}^{o}, \ldots, D_{\sigma(p)}^{o}\right)$ are ordered.

Finally, let us simply rewrite the previous theorem in the case where $\mu_{1}=\cdots=\mu_{p}$; it will be useful when we will try to interpret some conjectures:

Corollary 8. When $\mu$ is a CCI measure, then for all $p \geq 1$,

$$
\alpha\left(\mu^{\otimes p}\right)=\tilde{\xi}\left(\alpha(\mu)^{\otimes p}\right) .
$$

Remark. We could have derived Theorem 6 in the case $\lambda \geq 1$ (and therefore also Theorem 7 and Corollary 8) without using the strict concavity result (i.e. Lemma 4). More precisely, fix $\lambda^{\prime} \geq 1$, and choose $\lambda \geq 0$ such that $\tilde{\xi}(1, \lambda)=\lambda^{\prime}$. Define $Q(\delta)$ just as in the proof of Theorem 6. Equation (10) shows that

$$
Q(\delta) \approx \exp \left\{-\tilde{\xi}\left(\lambda^{\prime}, \alpha(\mu)\right) L(\delta)\right\} \text { when } \delta \rightarrow 0+.
$$

On the other hand,

$$
Q(\delta)=\mu\left[1_{\left\{D^{o} \in \mathcal{C}_{\delta}\right\}} v\left(\exp \left(-\lambda L^{+}(W)\right) 1_{\left\{W \in C_{\delta}, W \subset D^{+}\right\}}\right)\right] .
$$

But complete conformal invariance of $v$ shows that

$$
\nu\left(\exp \left(-\lambda L^{+}(W)\right) 1_{\left\{W \in C_{\delta}, W \subset D^{+}\right\}}\right) \approx \exp \left(-\lambda^{\prime} L^{+}(\gamma)\right) \text { when } \delta \rightarrow 0+.
$$

Hence (using simply the continuity of the mapping $\lambda \mapsto \tilde{\xi}(\alpha, \lambda)$ ), we get that

$$
Q(\delta) \approx \Psi^{+}\left(\lambda^{\prime}, L(\delta)\right) \text { when } \delta \rightarrow 0+
$$

so that eventually $\beta^{+}\left(\mu, \lambda^{\prime}\right)$ exists and is equal to $\tilde{\xi}\left(\lambda^{\prime}, \alpha(\mu)\right)$.

We prefered to give the general proof of Theorem 6 as we will use an analogous argument (involving strict concavity) in the next section. 


\section{Bridges in annuli}

\subsection{Conformal invariant measures in annuli}

Suppose that $\mu$ is a CCI measure on $\mathcal{C}^{o}$. As we have already seen, by conformally transforming the measures

$$
\mu_{V_{1, \delta}, V_{2, \delta}, D}
$$

we can obtain measures on oriented bridges connecting disjoint, connected subsets of boundaries of simply connected domains. One important family of such measures is the family of measures on the rectangles as in (1). In this section we show that we can also associate to $\mu$ a family of measures on bridges in annuli.

Let us first introduce some notation. For any $l>0$, we define the annulus $Q_{l}$,

$$
Q_{l}=\left\{r e^{i \theta}: \theta \in \mathbb{R}, r \in\left(1, e^{l}\right)\right\} .
$$

The boundary of $Q_{l}$ consists of the unit circle

$$
C_{0}=\left\{e^{i \theta}: \theta \in[0,2 \pi)\right\}
$$

and the outer circle

$$
C_{l}=\left\{e^{l+i \theta}: \theta \in[0,2 \pi)\right\} .
$$

We will consider measures on the set $\mathcal{Z}_{l}$ of connected compact subsets of $\bar{Q}_{l}$ that intersect both of these two circles.

Suppose $K \in \mathcal{Z}_{l}$ is simply connected. Then, $K$ does not disconnect 0 from infinity (there exists a continuous path from 0 to $C_{l}$ that does not intersect $K$ ) and there exists only one connected component of $\bar{Q}_{l} \backslash K$ that intersects both $C_{0}$ and $C_{l}$. We denote it by $\Delta^{\#}=\Delta^{\#}(K)$. Define also $\Delta^{o}(K)=\bar{Q}_{l} \backslash \Delta^{\#}$. When $K=\Delta^{o}(K)$, we say that $K$ is a bridge over $Q_{l}$. If $K$ is a bridge, then

$$
\Delta^{\#} \cap Q_{l}=Q_{l} \backslash K .
$$

By slight abuse of notation, we will also use $\Delta^{\#}$ to denote the open set $\Delta^{\#} \cap Q_{l}$. We let $\mathcal{Z}_{l}^{o}$ be the set of all bridges over $Q_{l}$. It is easy to check that a simply connected compact set $K$ in $\mathcal{Z}_{l}$ is a bridge over $Q_{l}$ if and only if $K \cap C_{0}$ and $K \cap C_{l}$ are non-empty connected arcs (single points are considered arcs).

Let $S_{l}$ be the infinite vertical strip

$$
S_{l}=\{z \in \mathbb{C}: 0<\Re(z)<l\},
$$


and let

$$
\begin{gathered}
\partial_{1}=\{i y: 0 \leq y \leq 2 \pi\} \\
\partial_{2}=\partial_{2}(l)=\{l+i y:-\infty<y<\infty\} .
\end{gathered}
$$

It is straightforward to show that

$$
L\left(\partial_{1}, \partial_{2} ; S_{l}\right)=\log l+O(1), \quad l \rightarrow \infty .
$$

For each $l$, there is a unique $\delta=\delta(l)$ such that there is a conformal transformation $\Psi_{l}$ taking $D$ to $S_{l}$ with

$$
\Psi_{l}\left(V_{1, \delta}\right)=\partial_{1}, \quad \Psi_{l}\left(V_{2, \delta}\right)=\partial_{2} .
$$

From (2) we see that

$$
\delta(l)=l^{-1 / 2}+O(1), \quad l \rightarrow \infty .
$$

Let $\mu$ be a CCI measure with crossing exponent $\alpha=\alpha(\mu)$. The measure $\mu$ (restricted to $\mathcal{C}_{\delta}$ ) gives a measure $\Psi_{l}(\mu)$ on bridges in $\bar{S}_{l}$ connecting $\partial_{1}$ to $\partial_{2}$. The exponential map takes these bridges to compact sets in $\mathcal{Z}_{l}$. We will use $\hat{\mu}_{l}$ to denote the measure on $\mathcal{Z}_{l}$ derived from $\mu$ in this way. Since the exponential map is not injective from $S_{l}$ to $Q_{l}$, the image compact set is not necessarily simply connected. Note that

$$
\hat{\mu}_{l}\left(\mathcal{Z}_{l}\right) \approx l^{-\alpha}, \quad l \rightarrow \infty .
$$

In particular, the total mass of the measure goes to zero slower than $e^{-a l}$ for all $a>0$.

We define the measure $\bar{\mu}_{l}$ on $\mathcal{Z}_{l}$ by

$$
\bar{\mu}_{l}=\hat{\mu}_{l} 1_{\left\{K \in \mathcal{Z}_{l}^{o}\right\}} .
$$

We can also view $\bar{\mu}_{l}, \hat{\mu}_{l}$ as families of measures on compact sets in cylinders of height $l$ (using the conformal correspondence between the annulus $Q_{l}$ and the cylinder $\left.C_{0} \times[0, l]\right)$.

We define the disconnection exponent $\bar{\alpha}=\bar{\alpha}(\mu)$ by

$$
\bar{\mu}_{l}\left(\mathcal{Z}_{l}\right)=\hat{\mu}_{l}\left(\mathcal{Z}_{l}^{o}\right) \approx e^{-l \bar{\alpha}}, \quad l \rightarrow \infty,
$$

in the case where such an $\bar{\alpha}$ exists.

Suppose $J \subset \bar{Q}_{l}$ is a simply connected region such that

$$
\partial_{0, J}=\partial J \cap C_{0},
$$

and

$$
\partial_{l, J}=\partial J \cap C_{l}
$$


are nontrivial connected arcs. Suppose also that $J$ does not disconnect the origin, i.e, that the origin is in the unbounded component of $\mathbb{C} \backslash \bar{J}$. There exist a unique $\delta=\delta(J)$ and a conformal transformation $\Phi$ from $D$ to $J$ with

$$
\Phi\left(V_{1, \delta}\right)=\partial_{0, J}, \quad \Phi\left(V_{2, \delta}\right)=\partial_{l, J} .
$$

This defines a measure $\Phi(\mu)$ on the set of $K \in \mathcal{Z}_{l}$ which stay in $J$. It is straightforward to check that this is the same measure as $\hat{\mu}_{l}$ restricted to bridges that go from $\partial_{0, J}$ to $\partial_{1, J}$ staying in $J$.

Example: Non-disconnecting Brownian crossings. In the case where the measure $\mu$ is $v$ (the measure corresponding to Brownian excursions), it is easy to see that the measure $\bar{\mu}_{l}$ is the measure corresponding to Brownian crossings of the annulus, restricted to those crossings that do not disconnect the origin. More precisely, pick $\theta_{0}$ uniformly on the unit circle. Consider a Brownian motion started from $r e^{i \theta_{0}}$ with $r>1$ until it exits the annulus $Q_{l}$. Keep only those paths that exit $Q_{l}$ through the outer circle $C_{l}$, and multiply the measure on paths by $(r-1)^{-1}$. When $r \rightarrow 1+$, this measure on paths converges to a measure on paths that can be interpreted as "Brownian crossings of $Q_{l}$ ". If we restrict to non-disconnecting crossings, and fill in the holes of their trace, we get the measure $\bar{\mu}_{l}$. We denote the bridges under this measure by $W=\Delta^{o}(W)$ and we let $W^{\#}=\Delta^{\#}(W)$. We also call the bridges the hulls of the Brownian crossings.

\subsection{Intersection exponents in annuli: Definition}

We will define the intersection exponent in annuli for ordered $p$-tuples of CCI measures. If $p=1$, we define the intersection exponent for annuli to be the disconnection exponent as in Sect. 4.1. Let $p \geq 2$ and suppose $\mu_{1}, \ldots, \mu_{p}$ are CCI measures. Suppose that

$$
\left(\Delta_{1}^{o}, \Delta_{1}^{\#}\right), \ldots,\left(\Delta_{p}^{o}, \Delta_{p}^{\#}\right)
$$

are chosen independently according to the measures $\bar{\mu}_{1, l}, \ldots, \bar{\mu}_{p, l}$ (associated respectively to $\left.\mu_{1}, \ldots, \mu_{p}\right)$. Let $\overline{\mathcal{O}}=\overline{\mathcal{O}}(p)$ be the event that the $p$ sets $\Delta_{1}^{o}, \ldots, \Delta_{p}^{o}$ are pairwise disjoint, and that they are ordered clockwise in $Q_{l}$.

We say that $\bar{\alpha}=\bar{\alpha}\left(\mu_{1}, \ldots, \mu_{p}\right)$ is the intersection exponent in annuli associated to the measures $\mu_{1}, \ldots, \mu_{p}$, if

$$
\otimes_{j=1}^{p} \bar{\mu}_{j, l}(\overline{\mathcal{O}}) \approx \exp \{-\bar{\alpha} l\}, \quad l \rightarrow \infty
$$

in the case where such an $\bar{\alpha}$ exists. 


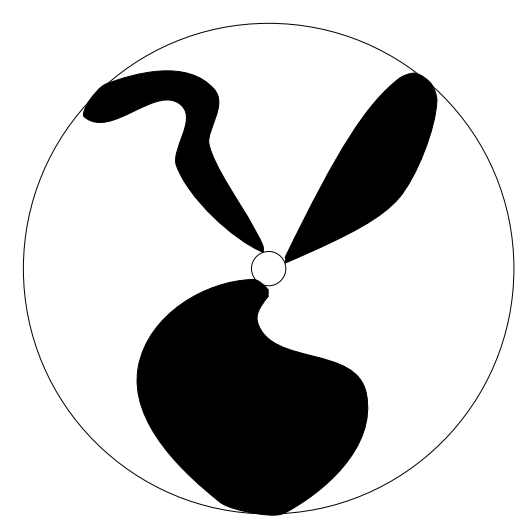

Fig. 4. Ordered bridges in the annulus

Note that

$$
\otimes_{j=1}^{p} \bar{\mu}_{j, l}(\overline{\mathcal{O}})=\otimes_{j=1}^{p} \hat{\mu}_{j, l}(\overline{\mathcal{O}})
$$

(if the sets do not intersect they necessarily do not disconnect the origin).

As in the previous section, it is easy to see that $\bar{\alpha}\left(\mu_{1}, \ldots, \mu_{p}\right)$ can be viewed as the disconnection exponent associated to a single CCI measure.

Lemma 9. Define $\mu=\Xi\left(\mu_{1}, \ldots, \mu_{p}\right)$ as at the end of Sect. 3.1. Then,

$$
\bar{\alpha}(\mu)=\bar{\alpha}\left(\mu_{1}, \ldots, \mu_{p}\right) .
$$

Proof. Define the sets $\left(\Delta_{1}^{o}, \ldots, \Delta_{p}^{o}\right)$ "independently" (i.e. with respect to the product measure) using the measures $\bar{\mu}_{1, l}, \cdots, \bar{\mu}_{p, l}$. Now, discard them if $\overline{\mathcal{O}}$ is not true. If $\overline{\mathcal{O}}$ is true, define the connected component $\Delta^{\#}$ of $\bar{Q}_{l} \backslash \cup_{j} \Delta_{j}^{o}$ such that

$$
\Delta_{1}^{o}, \ldots, \Delta_{p}^{o}, \Delta^{\#}
$$

are ordered clockwise around 0 . Let $\Delta^{o}=\bar{Q}_{l} \backslash \Delta^{\#}$. This defines a measure on bridges over the annulus $Q_{l}$. It is straightforward to check that this measure is precisely $\bar{\mu}_{l}$ where $\mu=\Xi\left(\mu_{1}, \ldots, \mu_{p}\right)$.

\subsection{Brownian intersection exponents in annuli}

In [27], we focused on the case where $\mu_{1}=v^{\cup n_{1}}, \ldots, \mu_{p}=v^{\cup n_{p}}$. Let us state some of the results that we derived in that paper.

- For any positive integers $n_{1}, \ldots, n_{p}$, the exponent $\bar{\alpha}\left(\mu_{1}, \ldots, \mu_{p}\right)$ is well-defined and is denoted by

$$
\xi\left(n_{1}, \ldots, n_{p}\right) .
$$


- There is a unique extension $\xi\left(\lambda_{1}, \ldots, \lambda_{p}\right)$ to nonnegative real $\lambda_{1}, \ldots, \lambda_{p}$ satisfying

$$
\lambda_{i}, \lambda_{i^{\prime}} \geq 1
$$

for some $i \neq i^{\prime}$ so that $\xi$ satisfies the cascade relation

$$
\xi\left(\lambda_{1}, \ldots, \lambda_{p}\right)=\xi\left(\lambda_{1}, \ldots, \lambda_{j}, \tilde{\xi}\left(\lambda_{j+1}, \ldots, \lambda_{p}\right)\right),
$$

for all $i \leq j \leq p-1$, and the symmetry relation

$$
\xi\left(\lambda_{\sigma(1)}, \ldots, \lambda_{\sigma(p)}\right)=\xi\left(\lambda_{1}, \ldots, \lambda_{p}\right) .
$$

Here $\sigma$ denotes a permutation of $\{1, \ldots, p\}$.

- There exists an increasing function $\eta:[\tilde{\xi}(1,1), \infty) \rightarrow \mathbb{R}_{+}$such that $\xi=\eta \circ \tilde{\xi}$.

Note (see [27]) that the exponent $\xi\left(n_{1}, n_{2}\right)$ is the intersection exponent for packs of Brownian motions in the plane as defined in the introduction. Let us recall how the exponents $\xi(1, \lambda)$ are defined in the present context. Suppose that $W=W^{o}$ is the hull of a non-disconnecting Brownian crossing under the measure $\bar{v}_{l}$ and let $W^{\#}=Q_{l} \backslash W$ as before. Define

$$
L^{\#}(W)=L\left(C_{0}, C_{l} ; W^{\#}\right) .
$$

Loosely speaking $e^{-L^{\#}(W)}$ measures how difficult it is for another Brownian motion to cross the annulus without touching $W$. Then, the exponent $\xi(1, \lambda)$ is characterized by the relation:

$$
\bar{v}_{l}\left(\exp \left\{-\lambda L^{\#}(W)\right\}\right) \approx \exp \{-l \xi(1, \lambda)\}, \quad l \rightarrow \infty .
$$

Schwarz's inequality implies that $\lambda \mapsto \xi(1, \lambda)$ is concave. One rigorously known value (see [21]) is $\xi(1,2)=\xi(2,1)=2$, and it is known that

$$
\xi(1,0)>0 \text { and } \xi(1, \lambda) \sim \lambda / 2 \text { when } \lambda \rightarrow \infty .
$$

This implies immediately the following.

Lemma 10. The only solution of

$$
\lambda=\xi(1, \lambda)
$$

for $\lambda \geq 0$ is $\lambda=2$.

In [24] it was shown that the function

$$
\lambda \longmapsto \xi(1, \lambda)
$$

is strictly concave. Hence the analogue to Lemma 4 holds for $\xi$ as well as $\tilde{\xi}$. 


\subsection{Intersection exponents in annuli: Universality}

Suppose that $\mu$ is a CCI measure, $l>0$, and that $K=\Delta^{o}(K)$ is defined under the measure $\bar{\mu}_{l}$. Let $K^{\#}=\Delta^{\#}(K)$ and let

$$
L^{\#}(K)=L\left(C_{0}(K), C_{l}(K) ; K^{\#}\right),
$$

where

$$
C_{0}(K)=C_{0} \cap \partial K^{\#} \text { and } C_{l}(K)=C_{l} \cap \partial K^{\#} .
$$

If $\lambda \geq 0$, the generalized intersection exponent in annuli (associated to $\mu) \bar{\beta}(\mu, \lambda)$ is defined by

$$
\bar{\mu}_{l}\left(\exp \left\{-\lambda L^{\#}(K)\right\}\right) \approx \exp \{-l \bar{\beta}(\mu, \lambda)\}, \quad l \rightarrow \infty,
$$

provided such a $\bar{\beta}(\mu, \lambda)$ exists. We are now ready to state the counterpart of Theorem 6 for annuli.

Theorem 11. For any CCI measure $\mu$, and for any $\lambda \geq 1$, the exponent $\bar{\beta}(\mu, \lambda)$ exists and

$$
\bar{\beta}(\mu, \lambda)=\xi(\alpha(\mu), \lambda) .
$$

Proof. We first introduce some notation. Suppose that $K$ is defined using the measure $\bar{\mu}_{l}$, and that $W$ is defined using the non-disconnecting Brownian crossing measure $\bar{v}_{l}$. We define $L^{\#}(K)$ and $L^{\#}(W)$ as before.

Suppose that $W \cap K \cap Q_{l}=\emptyset$. Note that this is equivalent to $K \cap Q_{l} \subset W^{\#}$ which is equivalent to $W \cap Q_{l} \subset K^{\#}$. Let $\Delta^{\#}(W, K)$ be the connected component of $K^{\#} \cap W^{\#}$ such that

$$
W, \Delta^{\#}(W, K), K
$$

are ordered counterclockwise in the annulus $Q_{l}$. Define also the corresponding extremal distance

$$
L^{\#}(W, K)=L\left(C_{0} \cap \partial \Delta^{\#}(W, K), C_{l} \cap \partial \Delta^{\#}(W, K) ; \Delta^{\#}(W, K)\right) .
$$

Suppose that $\lambda \geq 1$ is fixed. Let $\lambda^{\prime} \geq 0$ be defined by

$$
\tilde{\xi}\left(1, \lambda^{\prime}\right)=\lambda \text {. }
$$

We are going to study the asymptotic behavior as $l \rightarrow \infty$ of

$$
\bar{Q}(l)=\bar{\mu}_{l} \otimes \bar{v}_{l}\left(1_{\left\{W \cap K \cap Q_{l}=\emptyset\right\}} \exp \left\{-\lambda^{\prime} L^{\#}(W, K)\right\}\right) .
$$


Suppose that one first integrates with respect to $\bar{\mu}_{l}$. By conformal invariance, for fixed $W^{\#}$,

$$
\begin{aligned}
\bar{\mu}_{l}\left(1_{\left\{K \cap Q_{l} \subset W^{\#}\right\}} \exp \left\{-\lambda^{\prime} L^{\#}(W, K)\right\}\right) \\
=\mu\left(1_{\left\{D^{o} \in \mathcal{C}_{\delta}\right\}} \exp \left\{-\lambda^{\prime} L_{\delta}^{-}(\gamma)\right\}\right)
\end{aligned}
$$

where $\delta$ is chosen in such a way that $L(\delta)=L^{\#}(W)$. But this last quantity is logarithmically asymptotic to

$$
\exp \left\{-\beta\left(\mu, \lambda^{\prime}\right) L(\delta)\right\}=\exp \left\{-\beta\left(\mu, \lambda^{\prime}\right) L^{\#}(W)\right\}
$$

when $L^{\#}(W) \rightarrow \infty$ (and therefore also when $l \rightarrow \infty$ ). By Theorem 6 , $\beta\left(\mu, \lambda^{\prime}\right)=\tilde{\xi}\left(\alpha(\mu), \lambda^{\prime}\right)$. Hence,

$$
\begin{aligned}
\bar{Q}(l) & \approx \bar{v}_{l}\left(\exp \left\{-\tilde{\xi}\left(\alpha(\mu), \lambda^{\prime}\right) L^{\#}(W)\right\}\right) \\
& \approx \exp \left\{-l \xi\left(1, \tilde{\xi}\left(\alpha(\mu), \lambda^{\prime}\right)\right)\right\}
\end{aligned}
$$

when $l \rightarrow \infty$. From (11) and (12), we see that

$$
\xi\left(1, \tilde{\xi}\left(\alpha(\mu), \lambda^{\prime}\right)\right)=\xi\left(\alpha(\mu), \tilde{\xi}\left(1, \lambda^{\prime}\right)\right)=\xi(\alpha(\mu), \lambda) .
$$

Therefore,

$$
\bar{Q}(l) \approx \exp \{-l \xi(\alpha(\mu), \lambda)\}, \quad l \rightarrow \infty .
$$

Let us now return to the definition of $\bar{Q}_{l}$ and this time integrate first with respect to $\bar{v}_{l}$. When $K$ is fixed,

$$
\begin{aligned}
\bar{v}_{l}\left(1_{\left\{W \cap Q_{l} \subset K^{\#}\right\}} \exp \left\{-\lambda^{\prime} L^{\#}(W, K)\right\}\right) \\
=v\left(1_{\left\{W \in \mathcal{C}_{\delta}\right\}} \exp \left\{-\lambda^{\prime} L_{\delta}^{+}(W)\right\}\right)
\end{aligned}
$$

where $\delta$ is chosen in such a way that $L(\delta)=L^{\#}(K)$. But this quantity is logarithmically asymptotic to

$$
\exp \left\{-L^{\#}(K) \tilde{\xi}\left(1, \lambda^{\prime}\right)\right\}=\exp \left\{-L^{\#}(K) \lambda\right\}
$$

when $L^{\#}(K) \rightarrow \infty$ (and therefore also when $l \rightarrow \infty$ ). Hence, we finally see that there exists a function $\Theta(\cdot)$ such that

$$
\Theta(x) \approx \exp \{-\lambda x\}, x \rightarrow \infty
$$

such that

$$
\bar{\mu}_{l}\left(\Theta\left(L^{\#}(K)\right)\right) \approx \exp \{-l \xi(\alpha(\mu), \lambda)\}, l \rightarrow \infty .
$$

The analogue of Lemma 4 for $\xi$ can then be used to see that $\bar{\beta}(\mu, \lambda)$ exists and is equal to $\xi(\alpha(\mu), \lambda)$. 
Theorem 12. Suppose that $\mu_{1}, \ldots, \mu_{p}$ are CCI measures and that $p \geq 2$. Then, the intersection exponent $\bar{\alpha}\left(\mu_{1}, \ldots, \mu_{p}\right)$ exists and

$$
\left.\bar{\alpha}\left(\mu_{1}, \ldots, \mu_{p}\right)=\xi\left(\alpha\left(\mu_{1}\right), \ldots, \alpha\left(\mu_{p}\right)\right)\right) .
$$

In particular, for any CCI measure $\mu$ and any integer $p \geq 2$,

$$
\bar{\alpha}\left(\mu^{\otimes p}\right)=\xi\left(\alpha(\mu)^{\otimes p}\right) .
$$

Proof. Suppose that $\mu_{1}, \ldots, \mu_{p}$ are $p$ CCI measures; we have seen that $\alpha\left(\mu_{i}\right) \geq 1$ for all $i$. Because of the complete conformal invariance of the measures $\mu_{2}, \ldots, \mu_{p}$, one sees readily that

$$
\bar{\alpha}\left(\mu_{1}, \ldots, \mu_{p}\right)=\bar{\alpha}\left(\mu_{1}, \alpha\left(\mu_{2}, \ldots, \mu_{p}\right)\right) .
$$

From Corollary 7, we get that

$$
\bar{\alpha}\left(\mu_{1}, \ldots, \mu_{p}\right)=\xi\left(\alpha\left(\mu_{1}\right), \tilde{\xi}\left(\alpha\left(\mu_{2}\right), \ldots, \alpha\left(\mu_{p}\right)\right)\right),
$$

and using the cascade relations for $\xi$, we get immediately

$$
\bar{\alpha}\left(\mu_{1}, \ldots, \mu_{p}\right)=\xi\left(\alpha\left(\mu_{1}\right), \ldots, \alpha\left(\mu_{p}\right)\right) .
$$

The second equality in the theorem is just the first equality in the case where $\mu_{1}=\cdots=\mu_{p}=\mu$.

Note that the symmetry of $\xi$ implies the symmetry of $\bar{\alpha}$, i.e., under the same assumption as in (12), for any permutation $\sigma$ of $\{1, \ldots, p\}$,

$$
\bar{\alpha}\left(\mu_{1}, \ldots, \mu_{p}\right)=\bar{\alpha}\left(\mu_{\sigma(1)}, \ldots, \mu_{\sigma(p)}\right) .
$$

Just as for $\alpha$, this shows that $\bar{\alpha}$ is indeed the "unordered" intersection exponent in the sense

$$
\begin{aligned}
\otimes_{j=1}^{p} \bar{\mu}_{j, l}\left(\text { the sets } \Delta_{1}^{o}, \ldots, \Delta_{p}^{o} \text { are pairwise disjoint }\right) & \\
& \approx \exp \left\{-\bar{\alpha}\left(\mu_{1}, \ldots, \mu_{p}\right) l\right\}
\end{aligned}
$$

when $l \rightarrow \infty$.

\section{Conjectures and predictions}

In contrast to the rest of this paper, this section contains nonrigorous considerations. The goal of this section is to try to convince on an heuristic level that it is possible to interpret the conjectures for non-intersections between self-avoiding walks or percolation clusters in terms of non-intersections of Brownian paths. These models are believed to behave in a conformally invariant manner in the scaling limit. We do not give more details on what such a statement precisely means (it is actually not clear what the precise 
conjectures should be). One could expect that some well-chosen discrete measures on paths/sets will converge weakly in the scaling limit towards CCI measures (or at least measures that satisfy analogous conditions to CCI measures).

If this is the case, then our results (or our results generalized to the corresponding completely conformal invariance property) show that in the scaling limit, as what intersection exponents are concerned, these models behave like a generalized pack of Brownian excursions. This gives a nonrigorous justification to the "equivalence" between the various non-intersection exponents conjectures that we will present.

\subsection{Discrete excursion measure}

In this subsection, we point out some facts concerning the discrete counterpart to the Brownian excursion measure.

Suppose that $D$ is a fixed simply connected domain open bounded domain in $\mathbb{R}^{2}$. Consider $\epsilon>0$ and the set

$$
D_{\epsilon}=D \cap \epsilon \mathbb{Z}^{2}
$$

the set of points of the lattice $\epsilon \mathbb{Z}^{2}$ that belong to $D$. The discrete boundary of $D_{\epsilon}$ is simply

$$
\partial D_{\epsilon}=\Delta_{\epsilon}=\left\{z \in \epsilon \mathbb{Z}^{2}: \operatorname{dist}\left(z, D_{\epsilon}\right)=1\right\} .
$$

We say that the finite sequence $w=(w(0), \cdots, w(n))$ is an excursion in $D_{\epsilon}$ of length $n=n(w)$ if

$$
n \geq 1, w(0) \in \Delta_{\epsilon}, w(n) \in \Delta_{\epsilon},\{w(1), \ldots, w(n-1)\} \subset D_{\epsilon}
$$

and

$$
|w(1)-w(0)|=|w(2)-w(1)|=\cdots=|w(n)-w(n-1)|=\epsilon .
$$

Let $\Omega_{\epsilon}$ denote the set of such discrete excursions (with no condition on $n(w))$. Note that it is an infinite countable set. On $\Omega_{\epsilon}$ we define the measure $\mu_{\epsilon}$ as follows: For any $w \in \Omega_{\epsilon}$,

$$
\mu_{\epsilon}(\{w\})=\frac{1}{2 \pi 4^{n(w)}} .
$$

Note that there are no conditions on $w(0)$ and $w(n)$. In this discrete setting, "invariance under restriction of the discrete domain $D_{\epsilon}$ " of the measure is trivial: $\mu_{\epsilon}(w)$ is anyway either $\left(2 \pi 4^{n(w)}\right)^{-1}$ or zero (depending only on whether (13) holds or not). 
When the mesh $\epsilon$ of the grid tends to 0 , it is easy to see that this measure $\mu_{\epsilon}$ (or more precisely, the corresponding measure on suitably renormalized continuous paths) converges weakly towards the Brownian excursion measure $v^{\prime}$. Note that there is no need to renormalize the measure itself: When $\epsilon$ is small, and if $\partial D$ is smooth, the number of possible values for $w(0)$ explodes like $\epsilon^{-1}$ whereas the proportion of "macroscopic" excursions (say of diameter greater than some fixed $\delta$ ) decays like $\epsilon$, so that these two terms cancel out.

Hence, the Brownian excursion measures can be viewed as scaling limits of measures on discrete excursions. It has been proved (see [26] and the references therein) that intersection exponents for Brownian motions (the exponents $\xi$ described in the present paper) can also been viewed as intersection exponents between simple random walks, so that loosely speaking critical exponents are preserved by the invariance principle.

Duplantier-Kwon conjectured in [13] that

$$
\tilde{\xi}\left(1^{\otimes p}\right)=\frac{p(2 p+1)}{3}
$$

and

$$
\xi\left(1^{\otimes p}\right)=\frac{4 p^{2}-1}{12} .
$$

In [27], we proved that there exist two functions $U$ and $\eta$ such that for all $\lambda_{1}, \ldots, \lambda_{p} \geq 1$,

$$
\tilde{\xi}\left(\lambda_{1}, \ldots, \lambda_{p}\right)=U^{-1}\left(U\left(\lambda_{1}\right)+\ldots+U\left(\lambda_{p}\right)\right)
$$

and

$$
\xi\left(\lambda_{1}, \ldots, \lambda_{p}\right)=\eta \circ U^{-1}\left(U\left(\lambda_{1}\right)+\cdots+U\left(\lambda_{p}\right)\right) .
$$

These functional relations, the Duplantier-Kwon conjectures, and the cascade relations lead to the following conjectures (see again [27]):

$$
\begin{aligned}
& U(x)=\frac{\sqrt{24 x+1}-1}{\sqrt{24}} \\
& \eta(y)=\frac{(\sqrt{24 y+1}-1)^{2}-4}{48},
\end{aligned}
$$

and if we put $u_{i}=\sqrt{24 \lambda_{i}+1}-1$ (for $i \in\{1, \ldots, p\}$ ),

$$
\begin{aligned}
& \tilde{\xi}\left(\lambda_{1}, \ldots, \lambda_{p}\right)=\frac{\left(1+u_{1}+\cdots+u_{p}\right)^{2}-1}{24} \\
& \xi\left(\lambda_{1}, \ldots, \lambda_{p}\right)=\frac{\left(u_{1}+\cdots+u_{p}\right)^{2}-4}{48} .
\end{aligned}
$$


In particular,

$$
\begin{aligned}
\tilde{\xi}\left(\lambda^{\otimes p}\right) & =\frac{(1+p u)^{2}-1}{24} \\
\xi\left(\lambda^{\otimes p}\right) & =\frac{p^{2} u^{2}-4}{48}
\end{aligned}
$$

where $u=\sqrt{24 \lambda+1}-1$.

\subsection{Self-avoiding walks}

We now show how some well-known conjectures for self-avoiding walks in $\mathbb{Z}^{2}$ can be interpreted (nonrigorously) in terms of Brownian intersection exponents. A self-avoiding walk (SAW) in $\mathbb{Z}^{2}$ of $N$ steps is a nearest neighbor random walk path

$$
w=[w(0), \ldots, w(N)]
$$

with $w(0)=0$ and $w(j) \neq w(k), 0 \leq j<k \leq N$. We let $\Omega_{N}$ denote the set of SAWs of $N$ steps and $\mu_{N}$ the uniform probability measure on $\Omega_{N}$. It is well known (see [28] for details on SAWs) that

$$
\# \Omega_{N} \approx \beta^{N}, \quad N \rightarrow \infty
$$

where $\beta$ is the (lattice dependent) connective constant. It is generally believed that when $N \rightarrow \infty$ and in some appropriate scaling limit, the self-avoiding walk (more precisely, the uniform measure on SAWs) behaves (somewhat) in a conformally invariant manner and that exponents that describe the decay of non-intersection probabilities between independent self-avoiding walks are independent of the lattice used to define them. In particular, De Gennes [9] noticed that these critical exponents can be seen as limits when $N \rightarrow 0+$ of the exponents corresponding to the so-called $O(N)$ model. This has led to some general conjectures for the exact value of these exponents (see [30]).

For instance, it is believed that

$$
\# \Omega_{N} \asymp N^{\delta} \beta^{N}, N \rightarrow \infty,
$$

for some $\delta>0$. The exponent $\delta$ is believed to be lattice-independent (as long as it is a regular planar lattice) and can be viewed as an intersection exponent as it measures the decay of the probability $p_{N}$ that two self-avoiding walks of $N$ steps started from neighboring points do not intersect. If these two self-avoiding walks do not intersect, then they form (once glued together) a self-avoiding walk of $2 N$ steps. Hence,

$$
p_{N}=\frac{\# \Omega_{2 N}}{\left(\# \Omega_{N}\right)^{2}} \asymp \frac{1}{N^{\delta}}, N \rightarrow \infty .
$$


Conversely, a SAW at a point in its "middle" can be viewed as two mutually avoiding SAWs starting at that point. It has been predicted by Nienhuis (see e.g. [28]) that $\delta=11 / 32$.

Another exponent is the mean-squared displacement exponent $v$ defined by

$$
\mu_{N}\left[\left|\omega_{N}\right|^{2}\right] \approx N^{2 v}, \quad N \rightarrow \infty .
$$

This exponent can also be given in terms of the fractal dimension $d=$ $1 / \nu$. One expects that the paths of the continuum limit of SAW will have dimension $d$. Flory first predicted that $v=3 / 4$, i.e., that $d=4 / 3$, and this has been confirmed nonrigorously in a number of ways (see e.g. [28] for a review).

If $w^{1}, \cdots, w^{p}$ denote $p$ independent self-avoiding walks of $N$ steps started respectively from $(1,1), \cdots,(p, 1)$ under the uniform probability measure $P_{N}$, then an exponent $\kappa_{p}$ can be defined by

$$
P_{N}\left\{\forall 1 \leq i<j \leq p: w^{i}[0, N] \cap w^{j}[0, N]=\emptyset\right\} \approx N^{-\kappa_{p}}, \quad N \rightarrow \infty .
$$

Note that $\kappa_{2}=\delta$. An explicit prediction (see formula (20) below) has been given for $\kappa_{p}$ in terms of $p$ by Duplantier-Saleur [14]. Similarly, if $H$ denotes the upper half plane, the exponent $\tilde{\kappa}_{p}$ can be defined by

$$
\begin{gathered}
P_{N}\left\{\forall 1 \leq i<j \leq p: w^{i}[0, N] \cap w^{j}[0, N]=\emptyset\right. \text { and } \\
\left.\forall 1 \leq i \leq p, w^{i}[0, N] \subset H\right\} \approx N^{-\tilde{\kappa}_{p}}, \quad N \rightarrow \infty .
\end{gathered}
$$

An explicit formula for $\tilde{\kappa}_{p}$ in terms of $p$ has also been conjectured by Duplantier-Saleur [14] (see formula (21) below).

Suppose that the limit of self-avoiding walks gives a conformally invariant measure $\mu^{S A W}$ as in this paper with crossing exponent $\alpha$. Then a SAW corresponds to " $\alpha$ Brownian motions" for some $\alpha$. More precisely, consider a natural measure on self-avoiding excursions in a disc, and suppose that this measure converges to a conformally invariant measure $\mu^{S A W}$. Define

$$
\mu^{2 S A W}=\Xi\left(\mu^{S A W}, \mu^{S A W}\right)
$$

the measure on pairs of non-intersecting self-avoiding excursions. One could also instead assume the convergence of measures on discrete pairs of nonintersecting self-avoiding excursions towards a CCI measure $\mu^{2 S A W}$. Let

$$
\alpha^{2 S A W}=\alpha\left(\mu^{2 S A W}\right) .
$$

Note that if $\alpha^{S A W}=\alpha\left(\mu^{S A W}\right)$ then one expects $\alpha^{2 S A W}=\tilde{\xi}\left(\alpha^{S A W}, \alpha^{S A W}\right)$.

We conjecture that a typical SAW which goes a distance of about $e^{L}$ (and hence has about $N=e^{d L}$ steps) should have only a few points where the second coordinate is minimal. (If there are many such minima, there are 
many ways to form new SAWs with only two minima by attaching three bonds - i.e. "a tooth"- at one of the minima.) Hence, if we choose a random point on such a SAW, the probability that the second coordinate is minimal should be of order $e^{-d L}$. In other words, the probability that two mutually avoiding SAWs (chosen from the uniform distribution on mutually avoiding SAWs in the annulus of radius $e^{L}$ ) stay in the upper half plane should be of order $e^{-d L}$. But by conformal invariance, the measure of mutually avoiding paths in the annulus going from radius 1 to radius $e^{L}$ staying in the upper half plane should be the same as the exponent for crossing an $L \times \pi$ rectangle. Hence we get the conjecture

$$
\alpha^{2 S A W}=\eta\left(\alpha^{2 S A W}\right)+d .
$$

A second conjecture comes from considering

$$
\frac{\left|\bar{\Xi}_{L}\right|}{\left|\bar{\mu}_{L}^{2 S A W}\right|}
$$

where $\Xi=\Xi\left(\mu^{2 S A W}, v\right)$ and $v$ is the Brownian (simple random walk) excursion measure. We will use the following rigorous result in our heuristics.

Lemma 13. There exist $0<c_{1}<c_{2}<\infty$ such that the following is true. Let $A \subset \mathbb{Z}^{2}$ be a connected subset and $R$ its diameter, i.e.,

$$
R=\sup \{|x-y|, x, y \in A\} .
$$

Let $S$ be a simple random walk in $\mathbb{Z}^{2}$ started from $x \in \mathbb{Z}^{2}$ under the probability measure $\mathbf{P}^{x}$, and

$$
\tau=\tau_{A}=\inf \{t \geq 1: S(t) \in A\} .
$$

Then

$$
c_{1} \leq \sum_{x \in A} \mathbf{P}^{x}\left\{\tau \geq R^{2}\right\} \leq c_{2} .
$$

Proof. (sketch) By monotonicity it suffices to show that

$$
\sum_{x \in A} \sum_{j=R^{2}}^{2 R^{2}} \mathbf{P}^{x}\{\tau \geq j\} \asymp R^{2}
$$

and

$$
\sum_{x \in A} \sum_{j=R^{2} / 2}^{R^{2}} \mathbf{P}^{x}\{\tau \geq j\} \asymp R^{2} .
$$


These two are done similarly; we will consider the first. By reversing paths, we can see that

$$
\sum_{x \in A} \sum_{j=R^{2}}^{2 R^{2}} \mathbf{P}^{x}\{\tau \geq j\}=\sum_{y \in \mathbb{Z}^{2}} \mathbf{P}^{y}\left\{R^{2} \leq \tau \leq 2 R^{2}\right\} .
$$

The invariance principle and connectivity of the set $A$ can be used to show that there is a $c>0$ such that the number of points with

$$
\mathbf{P}^{y}\left\{R^{2} \leq \tau \leq 2 R^{2}\right\}>c
$$

is at least $c R^{2}$. It is not difficult to estimate the other terms so that

$$
\sum_{y \in \mathbb{Z}^{2}} \mathbf{P}^{y}\left\{R^{2} \leq \tau \leq 2 R^{2}\right\} \asymp R^{2}
$$

and proves the lemma.

It follows from Lemma 13, that if $A$ is a connected subset of $\mathbb{Z}^{2}$ of radius $R$ and a point $x$ is chosen from $A$ using the uniform distribution on $A$, then

$$
\mathbf{E}\left[\mathbf{P}^{x}\left\{\tau \geq R^{2}\right\}\right] \asymp \frac{1}{\# A} .
$$

(Here the expectation is over $x$.) Note that $R^{2}$ is the number of steps of a simple random walk needed to obtain a path of radius about $R$. Using this lemma as a guide, we conjecture that

$$
\frac{\left|\bar{\Xi}_{L}\right|}{\left|\bar{\mu}_{L}^{2 S A W}\right|} \approx e^{-d L}
$$

(Recall that the SAW in the annulus of radius $e^{L}$ has about $e^{d L}$ points.) In other words,

$$
\bar{\alpha}\left(\mu^{2 S A W}, v\right)=\bar{\alpha}\left(\mu^{2 S A W}\right)+d .
$$

But

$$
\bar{\alpha}\left(\mu^{2 S A W}, \nu\right)=\xi\left(\alpha^{2 S A W}, 1\right) .
$$

Hence we get the conjecture

$$
\xi\left(\alpha^{2 S A W}, 1\right)=\eta\left(\alpha^{2 S A W}\right)+d .
$$

Note that (16) and (17) imply

$$
\alpha^{2 S A W}=\xi\left(\alpha^{2 S A W}, 1\right) .
$$

If this is true, then Lemma 10 implies that

$$
\alpha^{2 S A W}=2 .
$$


This shows that the exponent for pairs of nonintersecting SAWs in a half plane should be the same as the exponent for pairs of (intersecting) Brownian motions in a half plane. Note that we have not used the conjectured values for any of the exponents in this (nonrigorous) argument. Also, combining this with (16) yields

$$
d=2-\eta(2) .
$$

Recall that we know rigorously ([22]) that the Hausdorff dimension of the outer frontier of a planar Brownian path is precisely $2-\eta(2)$, so that this confirms Mandelbrot's observation (via simulations, see [29]) on the close relationship between self-avoiding walks and Brownian frontiers. An extension of this argument even indicates that they have the same multifractal spectrum (via the correspondence derived in [23]).

We now assume the conjectures for the Brownian exponents, (14) and (15). Then

$$
d=2-\eta(2)=\frac{4}{3} \text {. }
$$

The relation $\alpha^{2 S A W}=\tilde{\xi}\left(\alpha^{S A W}, \alpha^{S A W}\right)=2$ implies that

$$
\alpha^{S A W}=\frac{5}{8}
$$

i.e, that SAWs should be considered as " $5 / 8$ of a Brownian motion". The interpretation of $\xi\left(\alpha^{\otimes p}\right)$ and $\tilde{\xi}\left(\alpha^{\otimes p}\right)$ as intersection exponents suggest that (we now write $\alpha=\alpha^{S A W}$ )

$$
\begin{aligned}
d \kappa_{p} & =\xi\left(\alpha^{\otimes p}\right)-p \xi(\alpha) \\
d \tilde{\kappa}_{k} & =\tilde{\xi}\left(\alpha^{\otimes p}\right)-p \tilde{\xi}(\alpha) .
\end{aligned}
$$

Using $\alpha=5 / 8$ we get the conjectures

$$
\begin{gathered}
\kappa_{p}=\frac{9 p^{2}-5 p-4}{64}, \\
\tilde{\kappa}_{p}=\frac{18 p^{2}+7 p}{64} .
\end{gathered}
$$

These last two conjectures are predictions made by $[7,14]$. In particular,

$$
\delta=\kappa_{2}=\frac{11}{32} .
$$

The problem with this heuristic argument is that we have shown that there is no CCI measure with $\alpha=5 / 8$. One possible way out is to focus only on the (CCI ?) measure $\mu^{2 S A W}$ given by the union of two mutually avoiding self-avoiding excursions in a domain (this one would have $\alpha^{2 S A W}=2$ ). 
Actually, it could be that the strict CCI property may not hold in the scaling limit and that there might be some additional multiplicative scaling factors after conformal mappings that depend on the density of the lattice (i.e., on the metric on the plane). However, we believe that the spirit of our method will be adaptable to the conformal invariance property that will hold for the scaling limits of SAW and that the formal identification between $\kappa_{p}, \tilde{\kappa}_{p}$ and the Brownian exponents makes sense.

\subsection{Percolation clusters}

Analogous arguments can be developed for percolation clusters. We will be very brief here. Consider planar bond percolation (see, e.g., [16]) in the square lattice at the critical probability $p=1 / 2$ (each bond is open with probability $1 / 2$ ). Then, define a measure on percolation clusters. More precisely, take a simply connected domain $D$ and define $D_{\epsilon}$ and $\partial D_{\epsilon}$ just as in Sect. 5.1. Perform critical bond percolation in $D_{\epsilon}$ (i.e. on the bonds such that at least one end is in $D_{\epsilon}$ ). Define for any simply connected "discrete bridge" $K \subset D_{\epsilon}$, the event $A(K)$ that the discrete outer boundary of the bridge $\partial K \cap D_{\epsilon}$ is the subset of an outer boundary of one single cluster and put

$$
\mu_{\epsilon}(K)=\mathbf{P}(A(K)) .
$$

This gives a measure on "discrete bridges" in $D_{\epsilon}$. One can expect that $\mu_{\epsilon}$ will converge (for some well-chosen topology) when $\epsilon \rightarrow 0+$ towards an infinite measure $\mu^{\text {perc }}$ on sets in $D$. A simple duality argument shows indeed that the mass of the set of macroscopic bridges will stay bounded. The conformal invariance conjectures for percolation (see e.g. [20] for a review and also [6]) show that it is natural to conjecture that this infinite measure will have a conformal invariance property. On the other hand, invariance under restriction of the domain in the discrete setting is a straightforward consequence of the definition (22) and the existence of the crossing exponent seems to follow from a submultiplicativity argument. Hence, we are led to conjecture that $\mu^{\text {perc }}$ is a CCI measure.

This leads to an interpretation of intersection exponents between clusters in terms of Brownian exponents. Note that if two independent such clusters do not intersect, then they can be viewed as two clusters belonging to the same percolation realization. We do not want to detail here the argument, but just in the way than for self-avoiding walks, one is led to the conjecture that it is possible to sum up all conjectures by the simple heuristic

$$
\text { "one percolation cluster }=\text { union of } 2 \text { Brownian paths" }
$$


i.e.

$$
\alpha\left(\mu^{\text {perc }}\right)=2
$$

and this is consistent with the predictions (see e.g. $[15,8,5]$ and the references therein). In particular, the fractal dimension (and the multifractal spectrum viewed from outside) of the outer frontier of a macroscopic percolation cluster is identical to that of the outer fontier of a planar Brownian path. Similarly, one can recover the intersection exponents predicted in $[15,8]$ between clusters.

\subsection{Concluding remarks}

The main common feature between self-avoiding walks, percolation and simple random walks is the fact that these are defined in a local way.

- The evolution of a simple random walk in a domain does not depend on where the boundary of the domain actually is. It has probability $1 / 4$ to move in any direction (if the lattice is $\epsilon \mathbb{Z}^{2}$ ) regardless of where the boundary is.

- The existence of a given percolation cluster in a domain depends only on the bonds of the lattice where this percolation cluster is; as the state of the different edges are independent, this shows that it is therefore independent of where the boundary of the domain is.

- A similar observation may hold for a well-chosen measure on selfavoiding excursions.

This leads to the prediction that if the discrete measures on paths corresponding to one of these models converge to a measure with a conformal invariance property then this measure will also have a strong conformal invariance property comparable to the CCI property described in the present paper.

It is clear that this type of locality property will fail for many other models in statistical physics, e.g., the Ising model and the uniform spanning tree model. For the latter which is closed related to the loop-erased random walk, Kenyon $[18,19]$ has recently derived rigorously the values of intersection exponents. This work confirms conjectures derived from nonrigorous conformal invariance assumptions [10,27]. The locality property does not hold for loop-erased random walks: A loop-erased random walk "feels" the boundary of the domain via its erased loops so that the exponents computed by Kenyon can not be interpreted in terms of Brownian exponents. It would be interesting to see whether some of the conformally invariant sets recently constructed by Schramm [33] satisfy such a locality property. 
More generally, one is led to the following general conjecture. For any measure on paths that combines a locality property and conformal invariance, then the Hausdorf dimension of its outer boundary (and its multifractal spectrum) is identical to that of the outer boundary of a Brownian path. In particular, the dimension $4 / 3$ is universal in that sense. From the perspective of conformal field theory, this locality property seems to correspond to "central charge 0" in the corresponding Virasoro algebra.

Acknowledgements. It is a pleasure to thank Bertrand Duplantier, Rick Kenyon and Oded Schramm for very useful discussions and comments.

\section{References}

1. L.V. Ahlfors (1973) Conformal Invariants, Topics in Geometric Function Theory. New York: McGraw-Hill

2. M. Aizenman (1996) The geometry of critical percolation and conformal invariance. Statphys 19 (Xiamen, 1995), 104-120

3. M. Aizenman, A. Burchard (1999) Hölder regularity and dimension bounds for random curves. Duke Math J. 99, 419-453

4. M. Aizenman, A. Burchard, C. Newman, D. Wilson (1999) Scaling limits for minimal and random spanning trees in two dimensions. Random Structures Algorithms 15, 317-367

5. M. Aizenman, B. Duplantier, A. Aharony (1999) Path crossing exponents and the external perimeter in 2D percolation. Phys. Rev. Lett. 83, 1359-1362

6. I. Benjamini, O. Schramm (1998) Conformal invariance of Voronoi percolation. Commun. Math. Phys. 197, 75-107

7. J.L. Cardy (1984) Conformal invariance and surface critical behavior. Nucl. Phys. B 240 (FS12), 514-532

8. J.L. Cardy (1998) The number of incipient spanning clusters in two-dimensional percolation. J. Phys. A 31, L105

9. P.G. De Gennes (1972) Exponents for the excluded volume method as derived by the Wilson method. Phys. Lett. 38A, 339-340

10. B. Duplantier (1992) Loop-erased self-avoiding walks in two dimensions: exact critical exponents and winding numbers. Physica A 191, 516-522

11. B. Duplantier (1998) Random walks and quantum gravity in two dimensions. Phys. Rev. Lett. 82, 5489-5492

12. B. Duplantier (1999) Two-dimensional copolymers and exact conformal multifractality. Phys. Rev. Lett. 82, 880-883

13. B. Duplantier, K.-H. Kwon (1988) Conformal invariance and intersection of random walks. Phys. Rev. Lett. 61, 2514-2517

14. B. Duplantier, H. Saleur (1986) Exact surface and wedge exponents for polymers in two dimensions. Phys. Rev. Lett. 57, 3179-3182

15. B. Duplantier, H. Saleur (1987) Exact determination of the percolation hull exponent in two dimensions. Phys. Rev. Lett. 58, 2325

16. G. Grimmett (1989), Percolation. Berlin, Heidelberg, New York: Springer

17. C. Itzykon, J.-M. Drouffe (1989) Statistical Field Theory, Vol. 2. Cambridge: Cambridge University Press

18. R. Kenyon (1998) Conformal invariance of domino tiling. Ann. Probab. (to appear)

19. R. Kenyon (1998) The asymptotic determinant of the discrete Laplacian. (preprint)

20. R. Langlands, Y. Pouillot, Y. Saint-Aubin (1994) Conformal invariance in twodimensional percolation. Bull. A.M.S. 30, 1-61 
21. G.F. Lawler (1991) Intersections of Random Ralks. Boston: Birkhäuser

22. G.F. Lawler (1996) The dimension of the frontier of planar Brownian motion. Electron. Commun. Prob. 1(5)

23. G.F. Lawler (1997) The frontier of a Brownian path is multifractal. (preprint)

24. G.F. Lawler (1998) Strict concavity of the intersection exponent for Brownian motion in two and three dimensions. Math. Phys. Electron. J. 4(5)

25. G.F. Lawler (2000) Strict concavity of the half plane intersection exponent for planar Brownian motion. Electron. J. Prob. 5(8)

26. G.F. Lawler, E.E. Puckette (1998) The intersection exponent for simple random walk. Combinatorics. Probab. Comput. (to appear)

27. G.F. Lawler, W. Werner (1998) Intersection exponents for planar Brownian motion. Ann. Probab. 27, 1601-1642

28. N. Madras, G. Slade (1993) The self-avoiding walk. Boston: Birkhäuser

29. B.B. Mandelbrot (1982) The fractal geomtery of nature. Freeman

30. B. Nienhuis (1983) Critical behavior of two-dimensional spin models and charge asymmetry in the Coulomb gas. J. Stat. Phys. 34, 731-761

31. C. Pommerenke (1992) Boundary Behaviour of Conformal Maps. Berlin, Heidelberg, New York: Springer

32. D. Revuz, M. Yor (1991) Continuous Martingales and Brownian Motion. Berlin, Heidelberg, New York: Springer

33. O. Schramm (1999) Scaling limits of loop-erased random walks and uniform spanning trees. Israel J. Math. (to appear) 